\title{
CARDINALITY ESTIMATION FOR RANDOM STOPPING SETS BASED ON POISSON POINT PROCESSES*
}

\author{
Nicolas Privault**
}

\begin{abstract}
We construct unbiased estimators for the distribution of the number of points inside random stopping sets based on a Poisson point process. Our approach is based on moment identities for stopping sets, showing that the random count of points inside the complement $\overline{\mathrm{S}}$ of a stopping set $\mathrm{S}$ has a Poisson distribution conditionally to $\mathrm{S}$. The proofs do not require the use of set-indexed martingales, and our estimators have a lower variance when compared to standard sampling. Numerical simulations are presented for examples such as the convex hull and the Voronoi flower of a Poisson point process, and their complements.
\end{abstract}

Mathematics Subject Classification. 60G55, 60D05, 60G40, 60G57, 60G48.

Received March 1, 2020. Accepted January 14, 2021.

\section{INTRODUCTION}

The probability distribution of the area of certain random domains constructed from Poisson distributed points has been studied via Gamma-type complementary theorems in [12]. More precise Gamma-type distribution results have been obtained in [21] for the volume content of stopping sets, which are random sets that carry over the notion of stopping time to set-indexed processes, see [13,21]. The proofs of [21], see also [6] and Theorem 10.4.8 in [19], rely on Laplace transform arguments and on the martingale property for set-indexed stochastic exponentials and martingales, see e.g. [10]. A different approach to the distribution of stopping sets has been developed in [18] using an anticipating Girsanov theorem for the underlying Poisson point process, instead of changes of intensities as in the above references. In [3], unbiased estimators have been constructed for the volume of the convex hull generated by a point process, which is the complement of a stopping set.

In this paper, we characterize the distribution of the number of points in stopping sets and their complements using moment identities for point processes, and we derive new unbiased estimators for those distributions. Given a Poisson point process with a finite and diffuse intensity measure $\sigma$ on a measure space $(X, \mathcal{B}(X), \sigma)$, we consider identities of the form

$$
\mathbb{P}(N(B)=n)=\frac{1}{n !} \mathbb{E}\left[\mathrm{e}^{-\sigma(B)}(\sigma(B))^{n}\right], \quad n \geq 0,
$$

\footnotetext{
* This research is supported by the Ministry of Education, Singapore, under its Tier 1 Grant MOE2018-T1-001-201 RG25/18.

Keywords and phrases: Stochastic geometry, Poisson point process, factorial moments, stopping sets, random convex hull, Voronoi tessellation.

Division of Mathematical Sciences, School of Physical and Mathematical Sciences, Nanyang Technological University, 21 Nanyang Link, Singapore 637371, Singapore.

** Corresponding author: nprivault@ntu.edu.sg
} 
for the probability distribution of the count $N(B)$ of Poisson points within a random subset $B$ of $X$, extending the formula

$$
\mathbb{P}(N(B)=n)=\mathrm{e}^{-\sigma(B)} \frac{(\sigma(B))^{n}}{n !}, \quad n \geq 0,
$$

which is known for deterministic $B \in \mathcal{B}(X)$ such that $\sigma(B)<\infty$.

Clearly, (1.1) cannot hold for any random set. For example, when $X=[0, T], T>0$, with $\sigma(\mathrm{d} x)=\mathrm{d} x$, taking $B_{m}:=\left[0, T_{m}\right]$ where $T_{m}, m \geq 1$, denotes the $m$ th jump time of the standard Poisson process $\left(N_{t}\right)_{t \in \mathrm{R}_{+}}$, we have $\mathbb{P}\left(N\left(B_{m}\right)=n\right)=\mathbf{1}_{\{n=m\}}$ and

$$
\begin{aligned}
\frac{1}{n !} \mathbb{E}\left[\mathrm{e}^{-\sigma\left(B_{m}\right)}\left(\sigma\left(B_{m}\right)\right)^{n}\right] & =\frac{1}{n !} \mathbb{E}\left[\mathrm{e}^{-\sigma\left(\left[0, T_{m}\right]\right)}\left(\sigma\left(\left[0, T_{m}\right]\right)\right)^{n}\right] \\
& =\frac{1}{n ! m !} \int_{0}^{\infty} \mathrm{e}^{-2 x} x^{n+m-1} \mathrm{~d} x \\
& =\frac{(n+m) !}{n ! m ! 2^{n+m}}, \quad n \geq 0,
\end{aligned}
$$

which does not match (1.2).

On the other hand, for the random set $B_{m}:=\left[0, \min \left(T, T_{m}\right)\right]$ and its complement $\bar{B}_{m}:=X \backslash B_{m}$, the probability

$$
\mathbb{P}\left(N\left(\bar{B}_{m}\right)=n\right)=\mathbb{P}(N([0, T])=n+m)=\mathrm{e}^{-T} \frac{T^{n+m}}{(n+m) !}, \quad n, m \geq 0
$$

matches the expected value

$$
\begin{aligned}
\frac{1}{n !} \mathbb{E}\left[\mathrm{e}^{-\sigma\left(\bar{B}_{m}\right)}\left(\sigma\left(\bar{B}_{m}\right)\right)^{n}\right] & =\frac{1}{n !} \mathbb{E}\left[\mathrm{e}^{-\sigma\left(\left(\min \left(T_{m}, T\right), T\right]\right)}\left(\sigma\left(\left(\min \left(T_{m}, T\right), T\right]\right)\right)^{n}\right] \\
& =\frac{1}{n !} \int_{0}^{T}(T-x)^{n} \frac{x^{m-1}}{(m-1) !} \mathrm{d} x \\
& =\mathrm{e}^{-T} \frac{T^{n+m}}{(n+m) !}
\end{aligned}
$$

as in (1.1).

We will show that (1.1) remains true for a large family of random sets $\overline{\mathrm{S}}:=X \backslash \mathrm{S}$ which are the complements of stable and non-increasing stopping sets $\mathrm{S}$ in $X$, see Definitions 3.1 and 3.3. More precisely, denoting by $\mathcal{F}_{\mathrm{S}}$ the sigma-algebra generated by the random stopping set S, see Definition 3.2, we show in Corollary 4.2 that such random sets $\mathrm{S}$ satisfy the relation

$$
\mathbb{P}\left(N(\overline{\mathrm{S}})=n \mid \mathcal{F}_{\mathrm{S}}\right)=\frac{1}{n !} \mathrm{e}^{-\sigma(\overline{\mathrm{S}})}(\sigma(\overline{\mathrm{S}}))^{n}, \quad n \geq 0,
$$

which implies (1.1) and provides an unbiased estimator of $\mathbb{P}(N(\overline{\mathrm{S}})=n)$. This also shows that, given $\mathcal{F}_{\mathrm{S}}$, the count of points $N(\overline{\mathrm{S}})$ in the complement $\overline{\mathrm{S}}$ of the stopping set $\mathrm{S}$ has the Poisson distribution with parameter $\sigma(\overline{\mathrm{S}})$, a fact already noted in the literature when $\overline{\mathrm{S}}$ is the convex hull of a Poisson point process, see e.g. [8], $[3,16]$.

Our approach to the proof of (1.3) relies on moment identities for Poisson and more general point processes, see $[15]-[4,9,17]$. In particular, we show in Section 3 that, when $\overline{\mathrm{S}}$ is the complement of a stopping set $\mathrm{S}$, the 
factorial moments of $N(\overline{\mathrm{S}})$ coincide with the moments of $\sigma(\overline{\mathrm{S}})$ given $\mathcal{F}_{\mathrm{S}}$, i.e.

$$
\mathbb{E}\left[N(\overline{\mathrm{S}})_{(n)} \mid \mathcal{F}_{\mathrm{S}}\right]=\sigma(\overline{\mathrm{S}})^{n}, \quad n \geq 1
$$

where $x_{(n)}:=x(x-1) \cdots(x-n+1)$ is the descending factorial, which implies

$$
\mathbb{E}\left[N(\overline{\mathrm{S}})_{(n)}\right]=\mathbb{E}\left[\sigma(\overline{\mathrm{S}})^{n}\right], \quad n \geq 1,
$$

see (4.1). For $n=2,(1.4)$ is the usual conditional variance identity

$$
\begin{aligned}
\operatorname{Var}\left[N(\overline{\mathrm{S}}) \mid \mathcal{F}_{\mathrm{S}}\right] & =\mathbb{E}\left[N(\overline{\mathrm{S}})_{(2)} \mid \mathcal{F}_{\mathrm{S}}\right]+\mathbb{E}\left[N(\overline{\mathrm{S}}) \mid \mathcal{F}_{\mathrm{S}}\right]\left(1-\mathbb{E}\left[N(\overline{\mathrm{S}}) \mid \mathcal{F}_{\mathrm{S}}\right]\right) \\
& =(\sigma(\overline{\mathrm{S}}))^{2}+\sigma(\overline{\mathrm{S}})(1-\sigma(\overline{\mathrm{S}})) \\
& =\sigma(\overline{\mathrm{S}})
\end{aligned}
$$

which has been applied in [3] to unbiased volume estimation when $\overline{\mathrm{S}}$ is the open convex hull of a Poisson point process.

From (1.5) we also obtain the probability generating function identity

$$
\mathbb{E}\left[(1+t)^{N(\overline{\mathrm{S}})} \mid \mathcal{F}_{\mathrm{S}}\right]=\mathrm{e}^{t \sigma(\overline{\mathrm{S}})}, \quad t \in(-2,0),
$$

see Proposition 4.1, which implies Relation (1.3) and yields the conditional moment generating function

$$
\mathbb{E}\left[\mathrm{e}^{\alpha N(\overline{\mathrm{S}})} \mid \mathcal{F}_{\mathrm{S}}\right]=\mathrm{e}^{\left(\mathrm{e}^{\alpha}-1\right) \sigma(\overline{\mathrm{S}})}, \quad \alpha \leq 0,
$$

which shows that the conditional cumulant $\kappa_{n}\left(N(\overline{\mathrm{S}}) \mid \mathcal{F}_{\mathrm{S}}\right)$ of order $n \geq 1$ of $N(\overline{\mathrm{S}})$ given $\mathcal{F}_{\mathrm{S}}$ is given by

$$
\kappa_{n}\left(N(\overline{\mathrm{S}}) \mid \mathcal{F}_{\mathrm{S}}\right)=\sigma(\overline{\mathrm{S}})
$$

extending (1.6) to $n \geq 3$.

In Section 5 we present numerical simulations that illustrate the results of Section 4, based on examples of complements of stopping sets such as annuli, convex hulls, and the Voronoi flower and cell, based on Poisson-Voronoi tessellations. Although our estimators are typically built from a single point process sample, their performance is measured by their mean square error, evaluated over a larger number of samples. Those simulations show that (1.3) has a lower variance than that of the standard sampling estimator.

Based on the results of Section 4, in Section 6 we construct an unbiased estimator of the form

$$
\mathbf{1}_{\{N(X) \leq n\}} \frac{(-1)^{n-N(X)}}{(n-N(X)) !}(\sigma(\overline{\mathrm{S}}))^{n-N(X)} \mathrm{e}^{\sigma(\overline{\mathrm{S}})}, \quad n \geq 0
$$

for the probability distribution $\mathbb{P}(N(\mathrm{~S})=n)$ of the count $N(\mathrm{~S})$ of points in a stopping set $\mathrm{S}$, see Corollary 4.4 , and we present related numerical estimates based on the above stopping set examples.

\section{Moments of Point Processes}

We start with a brief presentation of point processes admitting a Papangelou intensity, for use in the moment identities of Section 3. Let $X$ be a Polish space with Borel $\sigma$-algebra $\mathcal{B}(X)$, equipped with a finite non-atomic 
measure $\sigma(\mathrm{d} x)$. We let

$$
\Omega^{X}:=\{\omega \subset X: \#(A \cap \omega)<\infty \text { for all compact } A \in \mathcal{B}(X)\}
$$

denote the space of locally finite configurations on $X$, whose elements $\omega \in \Omega^{X}$ are identified with the Radon point measures $\omega=\sum_{x \in \omega} \delta_{x}$, where $\delta_{x}$ denotes the Dirac measure at $x \in X$ and $\omega(K) \in \mathrm{N} \cup\{\infty\}$ represents the cardinality of $K \cap \omega$. A point process is a probability measure $P$ on $\Omega^{X}$ equipped with the $\sigma$-algebra $\mathcal{F}$ generated by the topology of vague convergence. It can be characterized by its Campbell measure $C$ defined on $\mathcal{B}(X) \otimes \mathcal{F}$ by

$$
C(A \times B):=\mathbb{E}\left[\sum_{x \in A \cap \omega} \mathbb{1}_{B}(\omega \backslash\{x\})\right], \quad A \in \mathcal{B}(X), \quad B \in \mathcal{F},
$$

which satisfies the Georgii-Nguyen-Zessin [14] identity

$$
\mathbb{E}\left[\int_{X} u(x ; \omega) \omega(\mathrm{d} x)\right]=\mathbb{E}\left[\int_{\Omega^{X}} \int_{X} u(x ; \omega \cup x) C(\mathrm{~d} x, \mathrm{~d} \omega)\right],
$$

for all measurable processes $u: X \times \Omega^{X} \rightarrow \mathbf{R}$ such that both sides of (2.1) make sense. In Sections 2 and 3 we deal with point processes whose Campbell measure $C(\mathrm{~d} x, d \omega)$ is absolutely continuous with respect to $\sigma \otimes P$, i.e.

$$
C(\mathrm{~d} x, \mathrm{~d} \omega)=c(x ; \omega) \sigma(\mathrm{d} x) P(\mathrm{~d} \omega)
$$

where the density $c(x ; \omega)$ is called the Papangelou density. We will also use the random measure $\hat{\sigma}^{n}\left(d \mathfrak{x}_{n}\right)$ defined on $X^{n}$ by

$$
\hat{\sigma}^{n}\left(d \mathfrak{x}_{n}\right)=\hat{c}\left(\mathfrak{x}_{n} ; \omega\right) \sigma\left(\mathrm{d} x_{1}\right) \cdots \sigma\left(\mathrm{d} x_{n}\right),
$$

where $\mathfrak{x}_{n}=\left(x_{1}, \ldots, x_{n}\right) \in X^{m}$ and $\hat{c}\left(\mathfrak{x}_{n} ; \omega\right)$ is the compound Campbell density $\hat{c}: \Omega_{0}^{X} \times \Omega^{X} \longrightarrow \mathbf{R}_{+}$defined inductively on the set $\Omega_{0}^{X}$ of finite configurations in $\Omega^{X}$ by

$$
\hat{c}\left(\left\{x_{1}, \ldots, x_{n}, y\right\} ; \omega\right):=c(y ; \omega) \hat{c}\left(\left\{x_{1}, \ldots, x_{n}\right\} ; \omega \cup\{y\}\right), \quad n \geq 0,
$$

see Relation (1) in [9]. In particular, the Poisson point process with intensity $\sigma(\mathrm{d} x)$ is a point process with Campbell measure $C=\sigma \otimes P$ and $c(x ; \omega)=1$, and in this case the identity (2.1) becomes the Slivnyak-Mecke formula, see $[11,20]$.

In the sequel, we consider (possibly random) sets $A$ such that

$$
\left\{\omega \in \Omega^{X}: A(\omega) \subset K\right\} \in \mathcal{F}
$$

for all $K \in \mathcal{K}(X)$, where $\mathcal{K}(X)$ denotes the collection of (deterministic) compact subsets of $X$. For such random sets we let $N(A)(\omega)$ denote the cardinality of $\omega \cap A(\omega)$. We first consider the factorial moment $\mathbb{E}\left[N(A)_{(n)}\right]$, where $A$ is a (possibly random) measurable subset of $X$. We denote by $\varepsilon_{x}^{+}$the addition operator defined on random variables $F: \Omega^{X} \rightarrow \mathbf{R}$ as

$$
\varepsilon_{x}^{+} F(\omega):=F(\omega \cup\{x\}), \quad x \in X, \quad \omega \in \Omega^{X},
$$


and we use the notation

$$
\varepsilon_{\mathfrak{x}_{n}}^{+}:=\varepsilon_{x_{1}}^{+} \cdots \varepsilon_{x_{n}}^{+}, \quad \mathfrak{x}_{n}=\left(x_{1}, \ldots, x_{n}\right) \in X^{n} .
$$

Proposition 2.1. ([4], Prop. 2.1) Let $A$ be a random measurable subset of $X$. For all $n \geq 1$ and sufficiently integrable random variable $F$, we have

$$
\mathbb{E}\left[F N(A)_{(n)}\right]=\mathbb{E}\left[\int_{X^{n}} \varepsilon_{\mathfrak{x}_{n}}^{+}\left(F \mathbf{1}_{A^{n}}\left(x_{1}, \ldots, x_{n}\right)\right) \hat{\sigma}^{n}\left(\mathrm{~d} x_{1}, \ldots, \mathrm{d} x_{n}\right)\right] .
$$

Standard moment identities for the count $N(A)$ of process points within $A$ can be obtained as a consequence of factorial moment identities, see [17] for Poisson stochastic integrals and [9] for point processes with random integrands. By Proposition 2.1 and the relation

$$
x^{n}=\sum_{k=0}^{n} S(n, k) x_{(k)}
$$

where $S(n, k)$ denotes the Stirling number of the second kind, we find the moment identity

$$
\mathbb{E}\left[F(N(A))^{n}\right]=\sum_{k=0}^{n} S(n, k) \mathbb{E}\left[\int_{X^{k}} \varepsilon_{\mathfrak{x}_{k}}^{+}\left(F \mathbf{1}_{A^{k}}\left(\mathfrak{x}_{k}\right)\right) \hat{\sigma}^{k}\left(d \mathfrak{x}_{k}\right)\right],
$$

for the random set $A$, see Lemma 4.1 of [4]. As a consequence of Proposition 2.1 and the relation

$$
(1+t)^{x}=1+\sum_{n=1}^{\infty} \frac{t^{n}}{n !} x_{(n)},
$$

we also obtain the following corollary on the Probability Generating Function (PGF) of the count $N(A)$ of process points in a (random) set $A$.

Corollary 2.2. For $A$ a random set and $F$ a bounded random variable we have

$$
\mathbb{E}\left[F(1+t)^{N(A)}\right]=\mathbb{E}[F]+\sum_{k=1}^{\infty} \frac{t^{k}}{k !} \mathbb{E}\left[\int_{X^{k}} \varepsilon_{\mathfrak{x}_{k}}^{+}\left(F \mathbf{1}_{A^{k}}\left(x_{1}, \ldots, x_{k}\right)\right)(\omega) \hat{\sigma}^{k}\left(\mathrm{~d} x_{1}, \ldots, \mathrm{d} x_{k}\right)\right]
$$

$t \in(-2,0)$.

Corollary 2.2 and the relation

$$
\mathbb{E}\left[F \mathbf{1}_{\{N(A)=n\}}\right]=\frac{1}{n !} \frac{\partial^{n}}{\partial s^{n}} \mathbb{E}\left[F(1+s)^{N(A)}\right]_{\mid s=-1}, \quad n \geq 0,
$$

allows us to recover the distribution of the discrete random variable $N(A)$ in the next corollary.

Corollary 2.3. For $A$ a random set and $F$ a bounded random variable, we have

$$
\begin{aligned}
& \mathbb{E}\left[F \mathbf{1}_{\{N(A)=n\}}\right] \\
& =\frac{1}{n !} \sum_{k=0}^{\infty} \frac{(-1)^{k}}{k !} \mathbb{E}\left[\int_{X^{k+n}} \varepsilon_{\mathfrak{x}_{k+n}}^{+}\left(F \mathbf{1}_{A^{k+n}}\left(x_{1}, \ldots, x_{k+n}\right)\right)(\omega) \hat{\sigma}^{k+n}\left(\mathrm{~d} x_{1}, \ldots, \mathrm{d} x_{k+n}\right)\right],
\end{aligned}
$$

$n \geq 0$. 


\section{Moments OF STOPPING SETS}

In this section and the following ones, the measure $\sigma$ is assumed to be finite on $(X, \mathcal{B}(X))$. We recall the definition of stopping set, cf. [21] and Definition 2.27 page 335 of [13]. Given $K$ in the collection $\mathcal{K}(X)$ of compact subsets of $X$, let

$$
\mathcal{F}_{K}:=\sigma(\omega(U): U \subset K, \sigma(U)<\infty)
$$

denote the sigma-algebra generated by $\omega \mapsto \omega(U)$, with $U \subset K$ and $\sigma(U)<\infty$.

Definition 3.1. A random set $\mathrm{S}$ is called a stopping set if it is a.s. compact and satisfies

$$
\left\{\omega \in \Omega^{X}: \mathrm{S}(\omega) \subset K\right\} \in \mathcal{F}_{K} \quad \text { for all } K \in \mathcal{K}(X) .
$$

We refer to e.g. Definition 1 in [21] for the following definition of sigma-algebra generated by a stopping set.

Definition 3.2. Given S a stopping set, we consider the stopped sigma-algebra

$$
\mathcal{F}_{\mathrm{S}}:=\sigma\left(B \in \mathcal{F}: B \cap\left\{\omega \in \Omega^{X}: \mathrm{S}(\omega) \subset K\right\} \in \mathcal{F}_{K}, K \in \mathcal{K}(X)\right) .
$$

In addition to the stopping set property, we will need the following two conditions.

Definition 3.3. $\quad$ i) A stopping set $\mathrm{S}$ is said to be non-increasing if

$$
\mathrm{S}(\omega \cup\{x\}) \subset \mathrm{S}(\omega), \quad \omega \in \Omega^{X}, \quad x \in X .
$$

ii) A stopping set $\mathrm{S}$ is said to be stable if

$$
x \in \mathrm{S}(\omega) \Longrightarrow x \in \mathrm{S}(\omega \cup\{x\}), \quad \omega \in \Omega^{X}, \quad x \in X .
$$

The above monotonicity and stability conditions are satisfied by common examples of stopping sets, starting with deterministic compact subsets of $X$. Examples of random stopping sets include:

- the minimal closed ball $B_{m}$ centered at the origin and containing exactly $m \geq 1$ points,

- the closed complement $\mathrm{S}$ of the convex hull $\overline{\mathrm{S}}$ of a point process inside a convex subset of $\mathbf{R}^{d}$,

- the Voronoi flower S, which is the union of balls centered at the vertices of the Voronoi polygon that contain the point 0 and exactly two other process points,

see also $[6,7]$ for other examples of stopping sets, such as the Voronoi sausage or the Delaunay lunes.

The following lemma, which is needed for the proof of the next Proposition 3.5, is proved in appendix.

Lemma 3.4. Let $\mathrm{S}$ be a non-increasing stopping set. Then, for any $\mathcal{F}_{\mathrm{S}}$-measurable random variable $F(\omega)$ we have

$$
\varepsilon_{x}^{+} F(\omega)=F(\omega), \quad x \in \overline{\mathrm{S}}(\omega), \quad \omega \in \Omega^{X} .
$$

Letting $y \in X$ and taking $F:=\mathbf{1}_{\mathrm{S}}(y) \in \mathcal{F}_{\mathrm{S}}$, Lemma 3.4 shows that

$$
\left(\varepsilon_{x}^{+} \mathbf{1}_{\mathrm{S}}(y)\right)(\omega)=\mathbf{1}_{\mathrm{S}(\omega)}(y), \quad y \in X, \quad x \in \overline{\mathrm{S}}(\omega), \quad \omega \in \Omega^{X} .
$$

The next Proposition 3.5 is also proved in appendix. 
Proposition 3.5. The complement $\overline{\mathrm{S}}$ of a stable and non-increasing stopping set $\mathrm{S}$ fulfills the condition

$$
\varepsilon_{\mathfrak{x}_{n}}^{+}\left(\mathbf{1}_{\overline{\mathrm{S}}}\left(x_{1}\right) \cdots \mathbf{1}_{\overline{\mathrm{S}}}\left(x_{n}\right)\right)=\mathbf{1}_{\overline{\mathrm{S}}}\left(x_{1}\right) \cdots \mathbf{1}_{\overline{\mathrm{S}}}\left(x_{n}\right), \quad x_{1}, \ldots, x_{n} \in X, \quad n \geq 1 .
$$

By Proposition 3.5 we obtain the following consequences of Proposition 2.1, starting with the next factorial moment identity.

Proposition 3.6. Let $\overline{\mathrm{S}}$ be the complement of a stable and non-increasing stopping set $\mathrm{S}$. For all $n \geq 1$ we have

$$
\mathbb{E}\left[F N(\overline{\mathrm{S}})_{(n)}\right]=\mathbb{E}\left[\int_{\overline{\mathrm{S}}^{n}} \varepsilon_{x}^{+} F \hat{\sigma}^{n}\left(d \mathfrak{x}_{n}\right)\right]
$$

for $F$ a bounded random variable.

Similarly, from (2.2) we have

$$
\mathbb{E}\left[F(N(\overline{\mathrm{S}}))^{n}\right]=\sum_{k=0}^{n} S(n, k) \mathbb{E}\left[\int_{\overline{\mathrm{S}}^{k}} \varepsilon_{\mathfrak{x}_{k}}^{+} F \hat{\sigma}^{k}\left(d \mathfrak{x}_{k}\right)\right]
$$

In addition, by (3.6) the moments of stopping sets can also be expressed as

$$
\begin{aligned}
\mathbb{E}\left[N(\mathrm{~S})^{n}\right] & =\mathbb{E}\left[(N(X)-N(\overline{\mathrm{S}}))^{n}\right] \\
& =\sum_{k=0}^{n}(-1)^{k}\left(\begin{array}{l}
n \\
k
\end{array}\right) \mathbb{E}\left[(N(X))^{n-k}(N(\overline{\mathrm{S}}))^{k}\right] \\
& =\sum_{k=0}^{n}(-1)^{k}\left(\begin{array}{l}
n \\
k
\end{array}\right) \sum_{l=0}^{k} S(k, l) \mathbb{E}\left[\int_{\overline{\mathrm{S}}^{l}} \varepsilon_{x_{1}}^{+} \cdots \varepsilon_{x_{l}}^{+}(N(X))^{n-k} \hat{\sigma}^{l}\left(d \mathfrak{x}_{l}\right)\right] \\
& =\sum_{k=0}^{n}(-1)^{k}\left(\begin{array}{l}
n \\
k
\end{array}\right) \sum_{l=0}^{k} S(k, l) \mathbb{E}\left[(l+N(X))^{n-k} \hat{\sigma}^{l}\left(\overline{\mathrm{S}}^{l}\right)\right],
\end{aligned}
$$

where we took $F:=(N(X))^{n-k}$ in (3.6).

As a consequence of Proposition 3.6 and of Relation (2.3) we have the next extension of Corollary 2.2.

Corollary 3.7. Let $\overline{\mathrm{S}}$ denote the complement of a stable, non-increasing stopping set $\mathrm{S}$. The Probability Generating Function of $N(\overline{\mathrm{S}})$ satisfies

$$
\mathbb{E}\left[F(1+t)^{N(\overline{\mathrm{S}})}\right]=\sum_{k=0}^{\infty} \frac{t^{k}}{k !} \mathbb{E}\left[\int_{\overline{\mathrm{S}}^{k}} \varepsilon_{\mathfrak{x}_{k}}^{+} F \hat{\sigma}^{k}\left(\mathrm{~d} x_{1}, \ldots, \mathrm{d} x_{k}\right)\right],
$$

for $F$ a bounded random variable, $t \in(-2,0)$.

Multiple differentiation of (3.7) at $t=-1$ yields the distribution of $N(\overline{\mathrm{S}})$ as

$$
\mathbb{E}\left[F \mathbf{1}_{\{N(\overline{\mathrm{S}})=n\}}\right]=\frac{1}{n !} \sum_{k=0}^{\infty} \frac{(-1)^{k}}{k !} \mathbb{E}\left[\int_{\overline{\mathrm{S}}^{k+n}} \varepsilon_{\mathfrak{x}_{k+n}}^{+} F \hat{\sigma}^{k+n}\left(\mathrm{~d} x_{1}, \ldots, \mathrm{d} x_{k+n}\right)\right], \quad n \geq 0
$$


Corollary 3.8. Let $\overline{\mathrm{S}}$ be the complement of a stable and non-increasing stopping set $\mathrm{S}$. We have the conditional distribution

$$
\mathbb{P}\left(N(\overline{\mathrm{S}})=n \mid \mathcal{F}_{\mathrm{S}}\right)=\frac{1}{n !} \sum_{k=0}^{\infty} \frac{(-1)^{k}}{k !} \mathbb{E}\left[\hat{\sigma}^{k+n}\left(\overline{\mathrm{S}}^{k+n}\right) \mid \mathcal{F}_{\mathrm{S}}\right], \quad n \geq 0 .
$$

Proof. Taking $F$ to be $\mathcal{F}_{\mathrm{S}}$-measurable in (3.7)-(3.8), by Lemma 3.4 we have $\varepsilon_{x_{1}}^{+} \cdots \varepsilon_{x_{k}}^{+} F=F, x_{1}, \ldots, x_{k} \in \overline{\mathrm{S}}$, hence from Corollary 3.7 we find

$$
\mathbb{E}\left[F(1+t)^{N(\overline{\mathrm{S}})}\right]=\sum_{k=0}^{\infty} \frac{t^{k}}{k !} \mathbb{E}\left[F \hat{\sigma}^{k}\left(\overline{\mathrm{S}}^{k}\right)\right],
$$

which implies

$$
\mathbb{E}\left[(1+t)^{N(\overline{\mathrm{S}})} \mid \mathcal{F}_{\mathrm{S}}\right]=\sum_{k=0}^{\infty} \frac{t^{k}}{k !} \mathbb{E}\left[\hat{\sigma}^{k}\left(\overline{\mathrm{S}}^{k}\right) \mid \mathcal{F}_{\mathrm{S}}\right],
$$

$t \in(-2,0)$, and yields $(3.9)$ by multiple differentiation.

\section{Stopping Sets BASED ON Poisson Point PROCEsses}

In the remainder of this paper we specialize the results of Sections 2 and 3 to the setting of a Poisson point process having a finite diffuse intensity measure $\sigma$ on $(X, \mathcal{B}(X))$. In this case we have $c(x, \omega)=1$, $\hat{\sigma}^{n}\left(d \mathfrak{x}_{n}\right)=\sigma\left(\mathrm{d} x_{1}\right) \cdots \sigma\left(\mathrm{d} x_{n}\right)$, and for all compact disjoint subsets $K_{1}, \ldots, K_{n}$ of $X, n \geq 1$, the mapping $\omega \mapsto\left(\omega\left(K_{1}\right), \ldots, \omega\left(K_{n}\right)\right)$ is a vector of independent Poisson distributed random variables on $\mathrm{N}$ with respective parameters $\sigma\left(K_{1}\right), \ldots, \sigma\left(K_{n}\right)$. From (3.5), we have

$$
\mathbb{E}\left[N(\overline{\mathrm{S}})_{(n)} \mid \mathcal{F}_{\mathrm{S}}\right]=\sigma(\overline{\mathrm{S}})^{n}, \quad n \geq 0
$$

where $\overline{\mathrm{S}}$ the complement of a stable, non-increasing stopping set $\mathrm{S}$, hence the factorial moments of $N(\overline{\mathrm{S}})$ coincide with the moments of $\sigma(\overline{\mathrm{S}})$. From (2.3) and (4.1) we obtain the following result as in Corollary 3.7.

Proposition 4.1. Let $\overline{\mathrm{S}}$ be the complement of a stable and non-increasing stopping set $\mathrm{S}$. We have

$$
\mathbb{E}\left[(1+t)^{N(\overline{\mathrm{S}})} \mid \mathcal{F}_{\mathrm{S}}\right]=\mathrm{e}^{t \sigma(\overline{\mathrm{S}})}, \quad t \in(-2,0)
$$

From Proposition 4.1 we recover the distribution of $N(\overline{\mathrm{S}})$ as in Corollary 3.8.

Corollary 4.2. Let $\overline{\mathrm{S}}$ be the complement of a stable and non-increasing stopping set $\mathrm{S}$. We have

$$
\mathbb{P}\left(N(\overline{\mathrm{S}})=n \mid \mathcal{F}_{\mathrm{S}}\right)=\frac{\mathrm{e}^{-(\sigma(\overline{\mathrm{S}}))}}{n !}(\sigma(\overline{\mathrm{S}}))^{n}, \quad n \geq 0 .
$$

Corollary 4.2 shows in particular that, given the stopping set $\mathrm{S}$, the count $N(\overline{\mathrm{S}})$ is a Poisson random variable with intensity $\sigma(\overline{\mathrm{S}})$, see Theorem 3.1 of $[3,16]$, when $\mathrm{S}$ is the closed complement of the Poisson convex hull $\overline{\mathrm{S}}$.

In the remainder of this section we construct an estimator for the number of Poisson points inside a stopping set $\mathrm{S}$, using the information provided by $\overline{\mathrm{S}}$. The following result is a consequence of (3.8), and will be used for the construction of stopping set estimators. 
Lemma 4.3. Let $\overline{\mathrm{S}}$ be the complement of a stable and non-increasing stopping set $\mathrm{S}$. The distribution of $N(\overline{\mathrm{S}})$ and $N(\mathrm{~S})$ satisfies

$$
\mathbb{P}(N(\overline{\mathrm{S}})=n \text { and } N(\mathrm{~S})=l)=\frac{(-1)^{n}}{n !} \mathbb{E}\left[\frac{(-\sigma(\overline{\mathrm{S}}))^{l+n-N(X)}}{(l-N(X)) !} \mathbf{1}_{\{N(X) \leq l\}}\right]
$$

$l, n \geq 0$.

Proof. Applying (3.8) to $F=\mathbf{1}_{\{N(X)=l+n\}}$ with the relation

$$
\varepsilon_{\mathfrak{x}_{k+n}}^{+} \mathbf{1}_{\{N(X)=l+n\}}=\mathbf{1}_{\{N(X)=l-k\}}, \quad \mathfrak{x}_{k+n}=\left(x_{1}, \ldots, x_{k+n}\right) \in X^{k+n},
$$

we have

$$
\begin{aligned}
& \mathbb{P}(N(\overline{\mathrm{S}})=n \text { and } N(\mathrm{~S})=l)=\mathbb{E}\left[F \mathbf{1}_{\{N(\overline{\mathrm{S}})=n\}}\right] \\
& =\frac{1}{n !} \sum_{k=0}^{\infty} \frac{(-1)^{k}}{k !} \mathbb{E}\left[\int_{\overline{\mathrm{S}}^{k+n}} \varepsilon_{\mathfrak{x}_{k+n}}^{+} F \sigma^{k+n}\left(\mathrm{~d} x_{1}, \ldots, \mathrm{d} x_{k+n}\right)\right] \\
& =\frac{1}{n !} \sum_{k=0}^{\infty} \frac{(-1)^{k}}{k !} \mathbb{E}\left[\mathbf{1}_{\{N(X)=l-k\}} \int_{\overline{\mathrm{S}}^{k+n}} \sigma^{k+n}\left(\mathrm{~d} x_{1}, \ldots, \mathrm{d} x_{k+n}\right)\right] \\
& =\frac{1}{n !} \sum_{k=0}^{l} \frac{(-1)^{k}}{k !} \mathbb{E}\left[\mathbf{1}_{\{N(X)=l-k\}}(\sigma(\overline{\mathrm{S}}))^{k+n}\right], \quad l, n \geq 0 .
\end{aligned}
$$

From (4.3) we can recover the relation

$$
\begin{aligned}
\mathbb{P}(N(\overline{\mathrm{S}})=n) & =\sum_{l=0}^{\infty} \mathbb{P}(N(\overline{\mathrm{S}})=n \text { and } N(\mathrm{~S})=l) \\
& =\frac{(-1)^{n}}{n !} \mathbb{E}\left[(-\sigma(\overline{\mathrm{S}}))^{n} \sum_{l=0}^{\infty} \frac{(-\sigma(\overline{\mathrm{S}}))^{l-N(X)}}{(l-N(X)) !} \mathbf{1}_{\{N(X) \leq l\}}\right] \\
& =\frac{1}{n !} \mathbb{E}\left[(\sigma(\overline{\mathrm{S}}))^{n} \mathrm{e}^{-\sigma(\overline{\mathrm{S}})}\right]
\end{aligned}
$$

which also follows from (4.2).

On the other hand, Lemma 4.3 allows us to construct an unbiased estimator

$$
\mathbf{1}_{\{N(X) \leq l\}} \frac{(-\sigma(\overline{\mathrm{S}}))^{l-N(X)}}{(l-N(X)) !} \mathrm{e}^{\sigma(\overline{\mathrm{S}})}
$$

for the distribution $\mathbb{P}(N(\mathrm{~S})=l)$ of the number of points in a stopping set $\mathrm{S}$, as in the next corollary.

Corollary 4.4. Let $\mathrm{S}$ be a stable and non-increasing stopping set $\mathrm{S}(\omega)$. We have

$$
\mathbb{P}(N(\mathrm{~S})=l)=\mathbb{E}\left[\mathbf{1}_{\{N(X) \leq l\}} \frac{(-\sigma(\overline{\mathrm{S}}))^{l-N(X)}}{(l-N(X)) !} \mathrm{e}^{\sigma(\overline{\mathrm{S}})}\right], \quad l \geq 0 .
$$


Proof. By (4.3), we have

$$
\begin{aligned}
& \mathbb{P}(N(\mathrm{~S})=l)=\sum_{n=0}^{\infty} \mathbb{P}(N(\overline{\mathrm{S}})=n \text { and } N(\mathrm{~S})=l) \\
& =\mathbb{E}\left[\mathbf{1}_{\{N(X) \leq l\}} \frac{(-\sigma(\overline{\mathrm{S}}))^{l-N(X)}}{(l-N(X)) !} \sum_{n=0}^{\infty} \frac{(\sigma(\overline{\mathrm{S}}))^{n}}{n !}\right] \\
& =\mathbb{E}\left[\mathbf{1}_{\{N(X) \leq l\}} \frac{(-1)^{l-N(X)}}{(l-N(X)) !}(\sigma(\overline{\mathrm{S}}))^{l-N(X)} \mathrm{e}^{\sigma(\overline{\mathrm{S}})}\right] .
\end{aligned}
$$

In particular, we have $\mathbb{P}(N(\mathrm{~S})=0)=\mathbb{E}\left[\mathbf{1}_{\{N(X)=0\}} \mathrm{e}^{\sigma(\overline{\mathrm{S}})}\right]$,

$$
\mathbb{P}(N(\mathrm{~S})=1)=\mathbb{E}\left[\left(\mathbf{1}_{\{N(X)=1\}}-\mathbf{1}_{\{N(X)=0\}} \sigma(\overline{\mathrm{S}})\right) \mathrm{e}^{\sigma(\overline{\mathrm{S}})}\right],
$$

and

$$
\mathbb{P}(N(\mathrm{~S})=2)=\mathbb{E}\left[\left(\frac{1}{2} \mathbf{1}_{\{N(X)=0\}}(\sigma(\overline{\mathrm{S}}))^{2}-\mathbf{1}_{\{N(X)=1\}} \sigma(\overline{\mathrm{S}})+\mathbf{1}_{\{N(X)=2\}}\right) \mathrm{e}^{\sigma(\overline{\mathrm{S}})}\right] .
$$

\section{Distribution of Stopping Set COMPlements}

The simulations presented in this section and the next one use a Poisson point process with flat intensity $\lambda>0$, i.e. $\sigma(\mathrm{d} x)=\lambda \mathrm{d} x$, and are done with the R Spatstat package [2].

In this section we estimate the distribution $\mathbb{P}(N(\overline{\mathrm{S}})=n)$ of the number of Poisson points inside the complement $\overline{\mathrm{S}}$ of a stopping set $\mathrm{S}$ using both the standard sampling estimator $\mathbf{1}_{\{N(\overline{\mathrm{S}})=n\}}$ and the alternative estimator

$$
\mathbb{P}\left(N(\overline{\mathrm{S}})=n \mid \mathcal{F}_{\mathrm{S}}\right)=\frac{(\sigma(\overline{\mathrm{S}}))^{n}}{n !} \mathrm{e}^{-\sigma(\overline{\mathrm{S}})}
$$

obtained from Corollary 4.2. The performances of the estimators $\mathbf{1}_{\{N(\overline{\mathrm{S}})=n\}}$ and $(5.1)$ are compared via their respective variances given by $\mathbb{P}(N(\overline{\mathrm{S}})=n)(1-\mathbb{P}(N(\overline{\mathrm{S}})=n))$, and

$$
\frac{1}{n !^{2}} \mathbb{E}\left[(\sigma(\overline{\mathrm{S}}))^{2 n} \mathrm{e}^{-2 \sigma(\overline{\mathrm{S}})}\right]-(\mathbb{P}(N(\overline{\mathrm{S}})=n))^{2} .
$$

As (5.1) is clearly satisfied when $\mathrm{S}$ is deterministic, we only consider examples of random stopping sets S.

\subsection{Annuli in finite volume}

In this case, $X:=B(0, R)$ is the ball of radius $R>0$ centered at 0 in $\mathbf{R}^{d}$ and we consider the stable and non-increasing stopping set $\mathrm{S}:=B_{m}$ defined as the smallest closed ball centered at the origin and containing $m \geq 1$ process points in $\omega$ (see Fig. 1 ). 


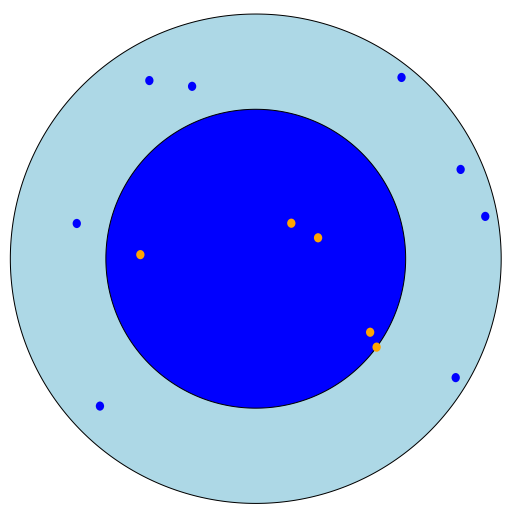

Figure 1. Sample disc $B_{m}$ (in blue) with $m=5$.

In this setting, the distribution of $N\left(\bar{B}_{m}\right)$ is explicitly known as

$$
\begin{aligned}
\mathbb{P}\left(N\left(\bar{B}_{m}\right)=n\right) & =\mathbb{P}\left(N(B(0, R))-N\left(B_{m}\right)=n\right) \\
& =\mathbb{P}(N(B(0, R))=n+m) \\
& =\mathrm{e}^{-\sigma(B(0, R))} \frac{(\sigma(B(0, R)))^{n+m}}{(n+m) !}, \quad n \geq 1,
\end{aligned}
$$

and

$$
\begin{aligned}
\mathbb{P}\left(N\left(\bar{B}_{m}\right)=0\right) & =\mathbb{P}\left(B_{m}=B(0, R)\right) \\
& =\mathbb{P}(N(B(0, R)) \leq m) \\
& =\mathrm{e}^{-\sigma(B(0, R))} \sum_{k=0}^{m} \frac{(\sigma(B(0, R)))^{k}}{k !}
\end{aligned}
$$

and we have the identity

$$
\mathbb{P}\left(\sigma\left(B_{m}\right)>r\right)=\mathbb{P}(N(B(0, r))<m)=\mathrm{e}^{-r} \sum_{k=0}^{m-1} \frac{r^{k}}{k !}, \quad 0 \leq r<R,
$$

which shows that the distribution of $\sigma\left(B_{m}\right)$ is given by

$$
\begin{aligned}
d \mathbb{P}\left(\sigma\left(B_{m}\right) \leq r\right) & =-d \mathbb{P}\left(\sigma\left(B_{m}\right)>r\right) \\
& =\mathbb{P}(N(B(0, R))<m) \delta_{R}(\mathrm{~d} r)+\mathrm{e}^{-r} \frac{r^{m-1}}{(m-1) !} \mathrm{d} r
\end{aligned}
$$

where $\delta_{R}(\mathrm{~d} r)$ denotes the Dirac measure at $R \in(0, \infty)$. In particular, it can be checked by closed form calculations that

$$
\mathbb{E}\left[\mathrm{e}^{t \sigma\left(\bar{B}_{m}\right)}\right]=\mathbb{E}\left[(1+t)^{N\left(\bar{B}_{m}\right)}\right], \quad t \in(-2,0),
$$




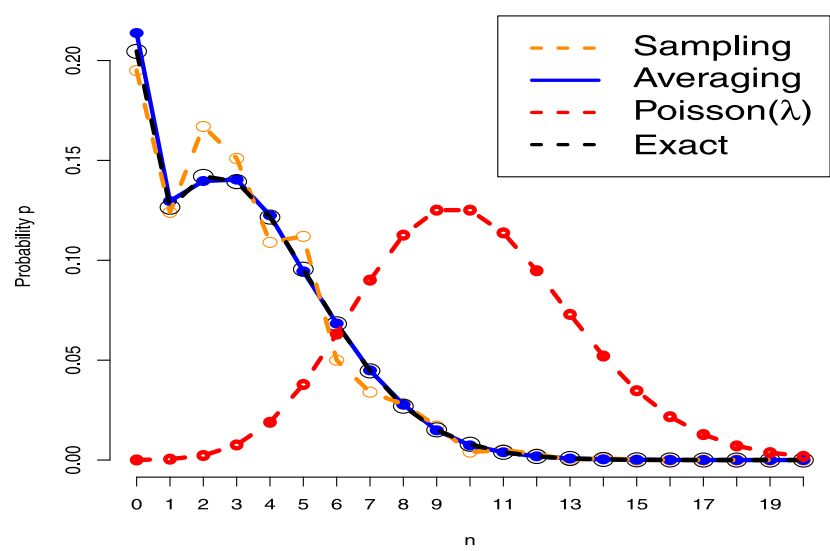

(A) Probability distribution

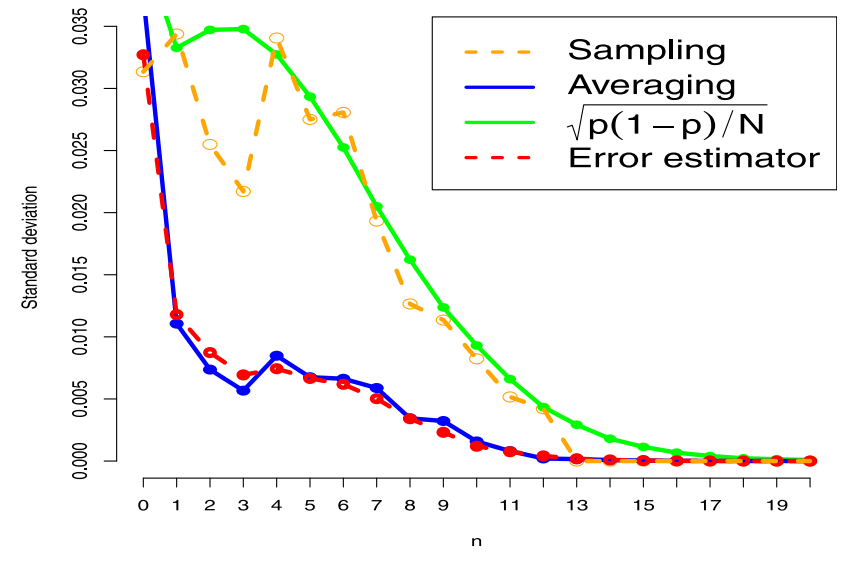

(B) Standard error

Figure 2. Distribution and standard error for $\bar{B}_{5}$, with $N=1000$ and $\lambda=10$.

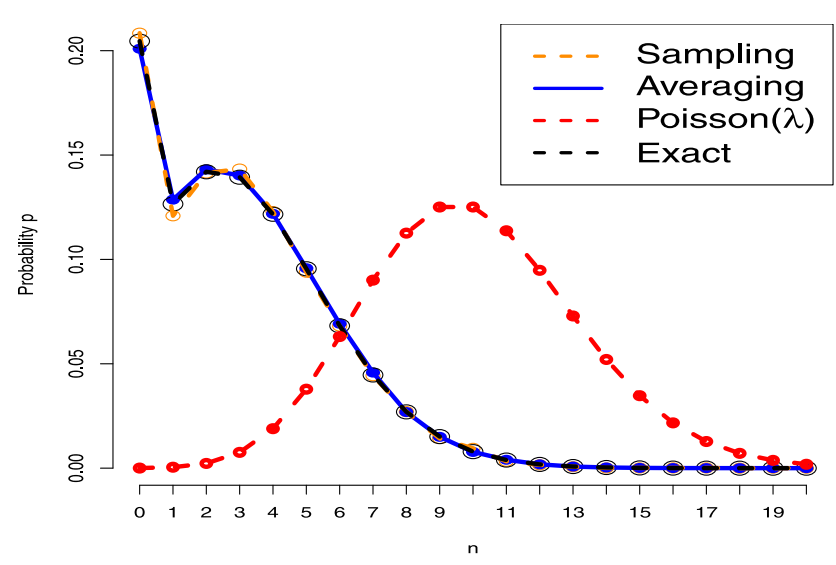

(A) Probability distribution

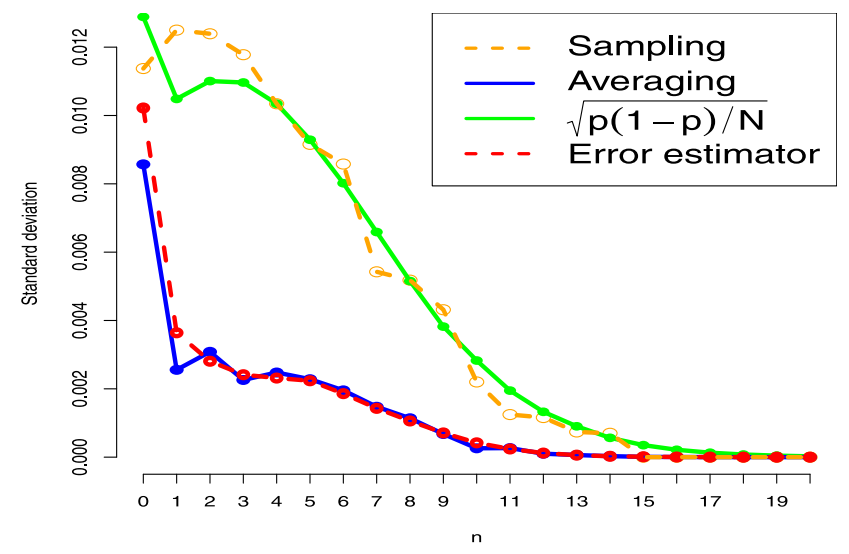

(B) Standard error

Figure 3 . Distribution and standard error for $\bar{B}_{5}$, with $N=10,000$ and $\lambda=10$.

as in Proposition 4.1, and

$$
\mathbb{P}\left(N\left(\bar{B}_{m}\right)=n\right)=\frac{1}{n !} \mathbb{E}\left[\left(\sigma\left(\bar{B}_{m}\right)\right)^{n} \mathrm{e}^{-\sigma\left(\bar{B}_{m}\right)}\right], \quad n \geq 1,
$$

in agreement with Corollary 4.2.

Using the estimator $\mathbf{1}_{\{N(\overline{\mathrm{S}})=n\}}$ ("Sampling") and the alternative estimator (5.1) ("Averaging"), the following simulations provide estimates of the distribution $\mathbb{P}\left(N\left(\bar{B}_{5}\right)=n\right)$ of the count of points strictly inside the convex hull $\bar{B}_{5}$ complement of $B_{5}$ in $X=B(0,1 / 2)$. Figures 2 and 3 are plotted with $N=1000$ and $N=10,000$ Monte Carlo samples respectively, together with the exact estimates (5.3)-(5.4) and the Poisson probability function with parameter $\lambda>0$.

The standard errors plotted in Figure 2b show that the estimator (5.1) ("Averaging") is more accurate and has a lower variance than the standard estimator $\mathbf{1}_{\{N(\overline{\mathrm{S}})=n\}}$ ("Sampling"). In this figure and the following ones, error estimates are provided in two different forms: 


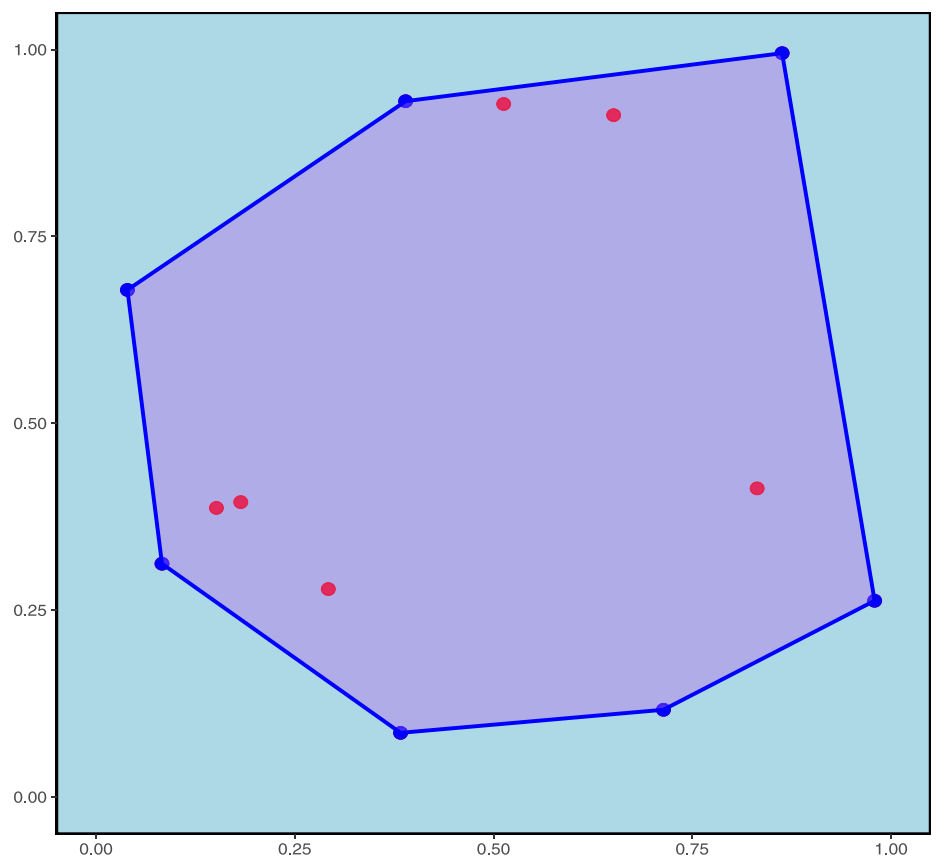

Figure 4. Sample convex hull of a Poisson point process.

- Monte Carlo error estimates for the standard estimator $\mathbf{1}_{\{N(\overline{\mathrm{S}})=n\}}$ ("Sampling") together with the corresponding error estimator

$$
\sqrt{\mathbb{P}(N(\overline{\mathrm{S}})=n)(1-\mathbb{P}(N(\overline{\mathrm{S}})=n)) / N},
$$

where $N$ is the number of Monte Carlo samples;

- Monte Carlo error estimates for (5.1) ("Averaging"), together with the corresponding estimator (5.2) ("Error estimator"), which can be computed as

$$
\begin{aligned}
& \sqrt{\frac{1}{n !^{2}} \mathbb{E}\left[(\sigma(\overline{\mathrm{S}}))^{2 n} \mathrm{e}^{-2 \sigma(\overline{\mathrm{S}})}\right]-(\mathbb{P}(N(\overline{\mathrm{S}})=n))^{2}} \\
& =\sqrt{\frac{1}{n !^{2} 2^{2 n}} \mathbb{E}\left[(2 \sigma(\overline{\mathrm{S}}))^{2 n} \mathrm{e}^{-2 \sigma(\overline{\mathrm{S}})}\right]-(\mathbb{P}(N(\overline{\mathrm{S}})=n))^{2}} \\
& =\sqrt{\frac{(2 n) !}{n !^{2} 2^{2 n}} \mathbb{P}(N(2 \overline{\mathrm{S}})=2 n)-(\mathbb{P}(N(\overline{\mathrm{S}})=n))^{2}}
\end{aligned}
$$

by applying (5.1).

\subsection{Open convex hull of a Poisson point process}

The closed complement $\mathrm{S}=\bar{C}$ of the (open) convex hull $\overline{\mathrm{S}}=C$ of a Poisson point process in a convex domain $X$ of finite intensity measure in $\mathbf{R}^{d}$ is a stable and non-increasing stopping set, see Section 3 , and Figure 4 for an illustration. The study of the convex hull of a random set of points is a classical topic in computational geometry, with numerous applications in statistics and computing, see e.g. [1] and references therein. 


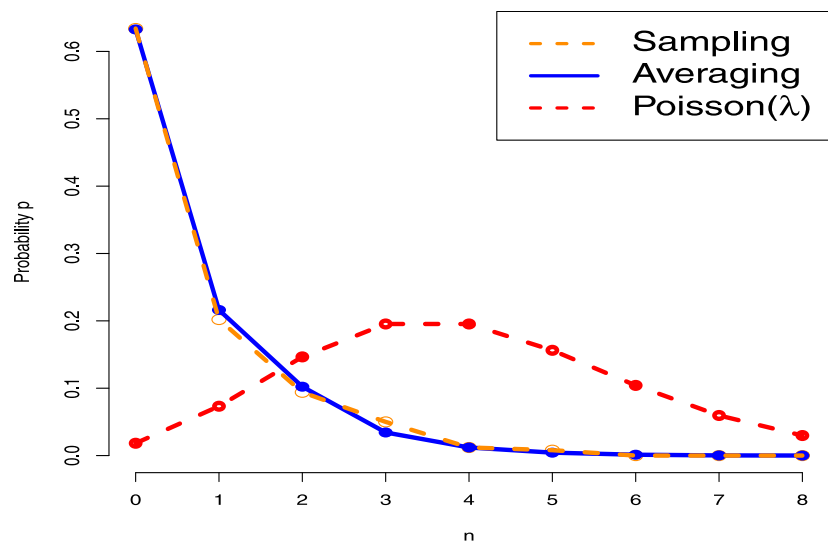

(A) Probability distribution

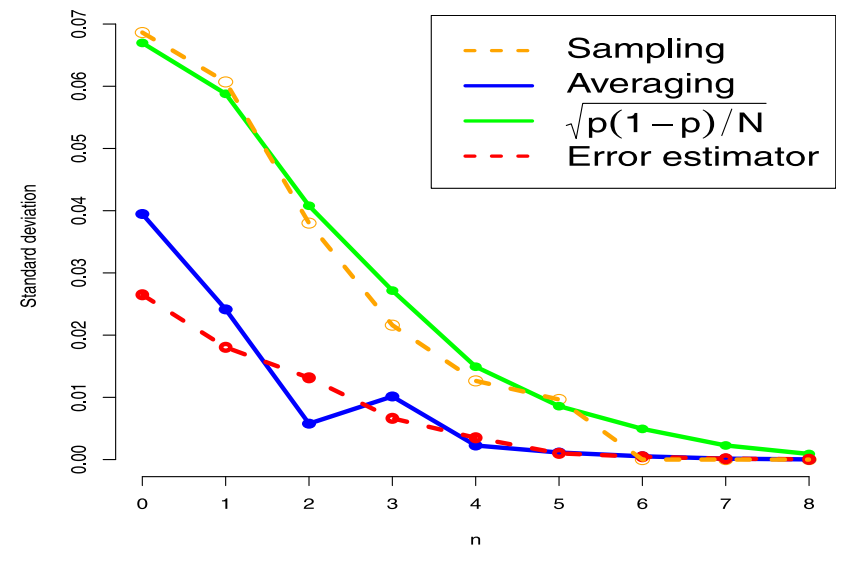

(B) Standard error

Figure 5. Distribution and standard error for the inside of the Poisson convex hull, $\lambda=4$.

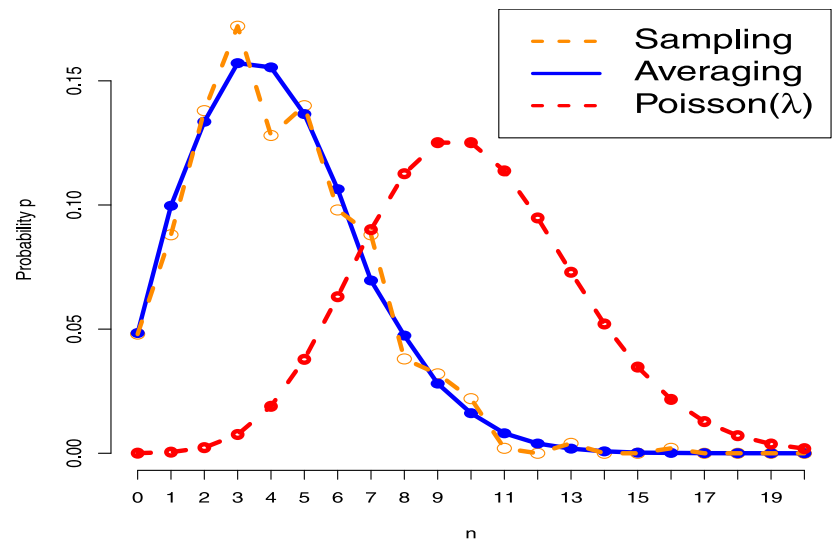

(A) Probability distribution

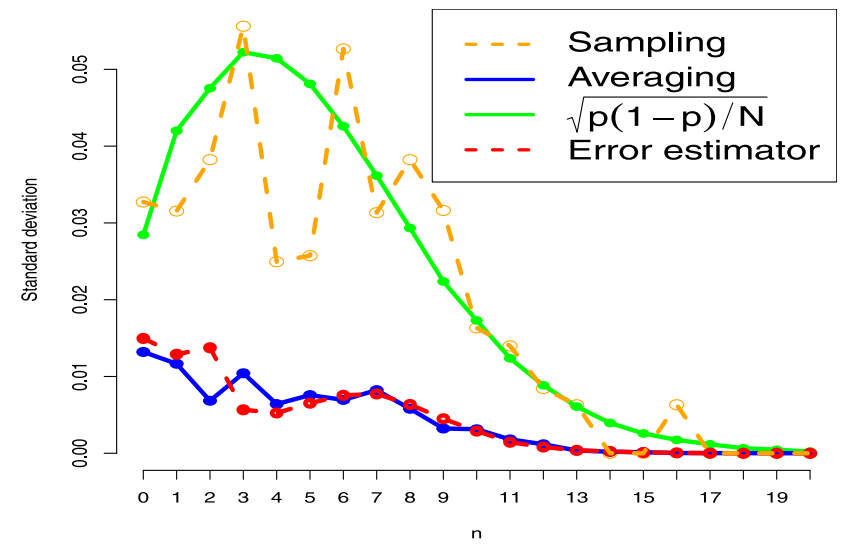

(B) Standard error

Figure 6. Distribution and standard error for the inside of the Poisson convex hull, $\lambda=10$.

In Figures 5 and 6 we provide estimates for the distribution $\mathbb{P}(N(\overline{\mathrm{S}})=n)$ of the count of points strictly inside the convex hull $\overline{\mathrm{S}}$ complement of $\mathrm{S}$, generated by the Poisson point process on $X=[0,1]^{2}$, which are plotted with $N=500$ Monte Carlo samples.

As in Figures 2-3, we check that the estimator (5.1) ("Averaging") is more accurate, as it has a lower variance than standard sampling when estimating the count of points in the complement $\overline{\mathrm{S}}$ of the Poisson convex hull S for two different values of the Poisson intensity parameter $\lambda$.

The estimates are plotted together with the Poisson probability function with parameter $\lambda>0$ as in Figures 2 and 3. The Monte Carlo error estimates "Sampling" and "Averaging" are respectively complemented with their estimators (5.2) ("Error estimator") and (5.5).

\subsection{Voronoi flower complement}

We consider the stopping set given by the Voronoi flower $\mathrm{S}$ based on a typical cell containing the point $(1 / 2,1 / 2)$ in the unit square $X=[0,1] \times[0,1]$, see Section 3 , up to a translation of the Poisson point process 


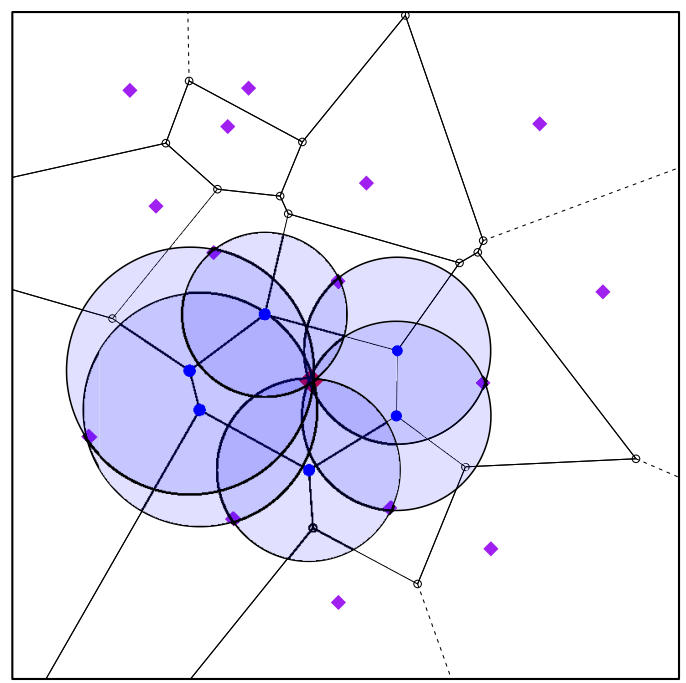

FiguRE 7. Sample Voronoi flower S.

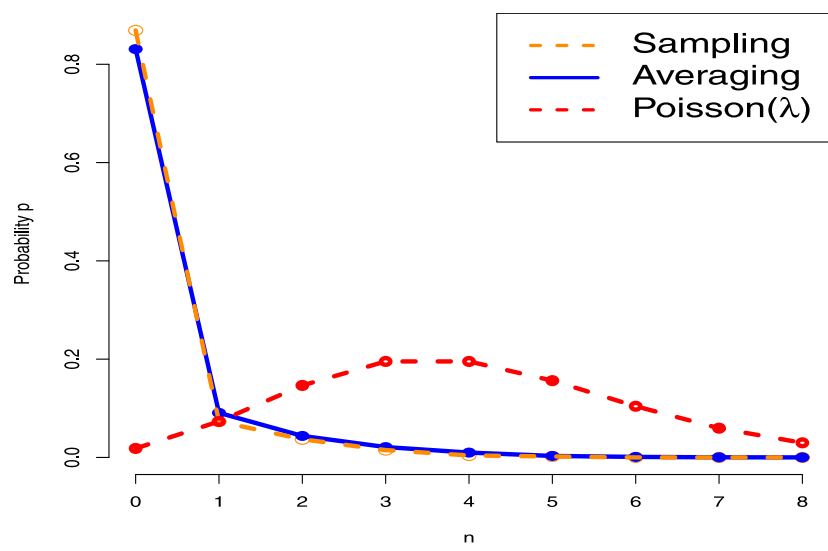

(A) Probability distribution

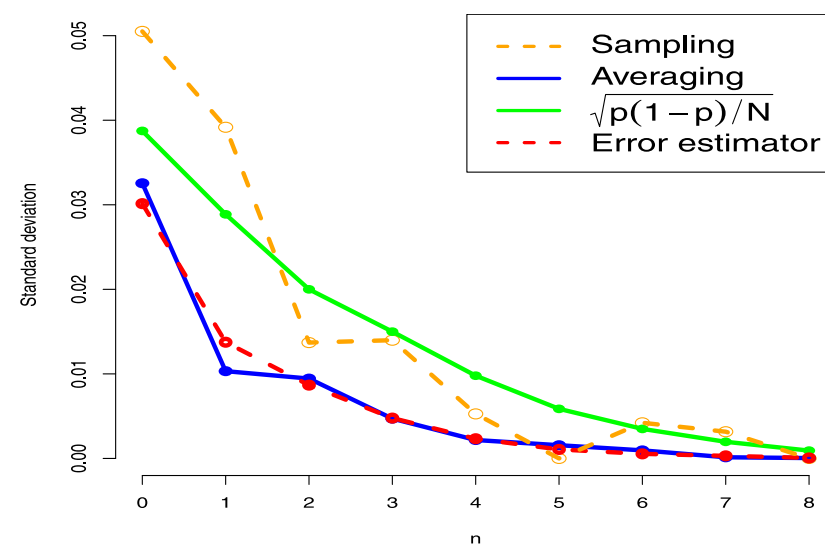

(B) Standard error

Figure 8. Distribution and standard error for the Voronoi flower complement, $\lambda=4$.

with flat intensity $\lambda>0$ (see Fig. 7). In case the window $X=[0,1] \times[0,1]$ does not contain any Voronoi cell around the point $(1 / 2,1 / 2)$ we let $\mathrm{S}=[0,1] \times[0,1]$, which is the case in particular when $N(X) \leq 3$.

Similarly to Figures 5 and 6 , the next simulations provide estimates for the distribution $\mathbb{P}(N(\bar{S})=n)$ of the count of points in the complement $\overline{\mathrm{S}}$ of the Voronoi flower $\mathrm{S}$ around the point $(1 / 2,1 / 2)$, generated by a Poisson point process with flat intensity $\lambda>0$ on the unit square $X=[0,1]^{2}$.

In Figures 8-10, which are plotted with $N=1000$ Monte Carlo samples, we also check that the estimator (5.1) ("Averaging") has lower variance than the standard sampling estimator $\mathbf{1}_{\{N(\overline{\mathrm{S}})=n\}}$ when estimating the count of points in the complement $\overline{\mathrm{S}}$ of the Voronoi flower $\mathrm{S}$ for two different values of the Poisson intensity parameter $\lambda$.

As in Figures 5 and 6, the Monte Carlo error estimates are respectively complemented with their estimators (5.2) ("Error estimator") and (5.5). 


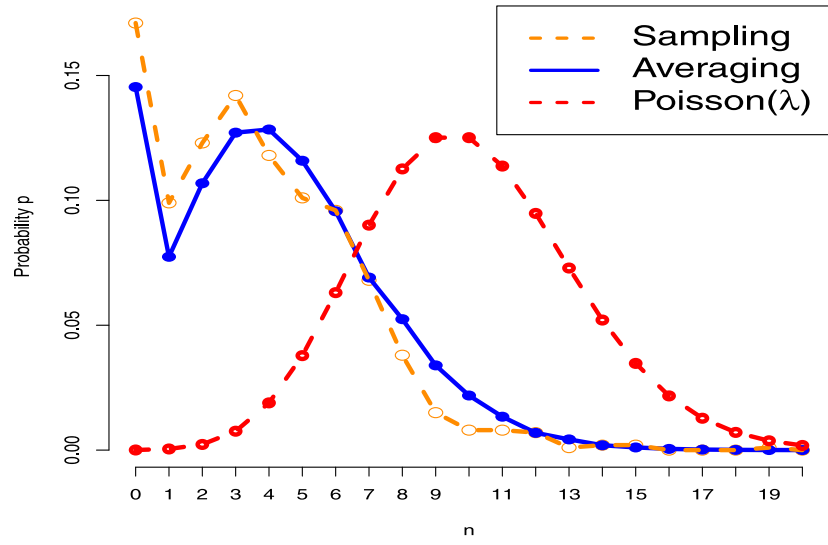

(A) Probability distribution

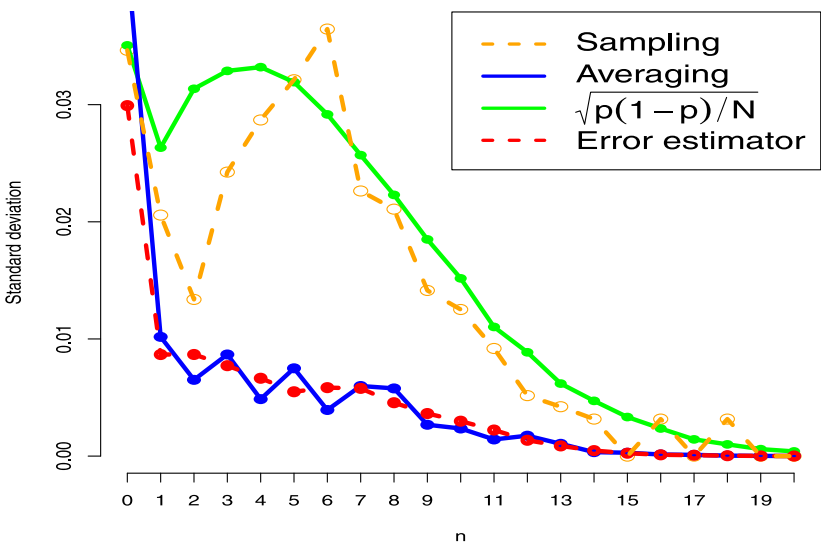

(B) Standard error

Figure 9. Distribution and standard error for the Voronoi flower complement, $\lambda=10$.

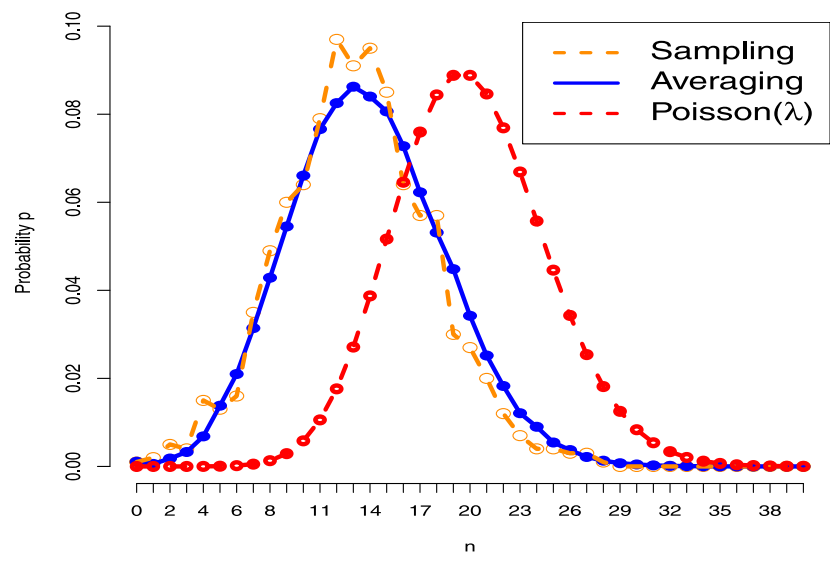

(A) Probability distribution

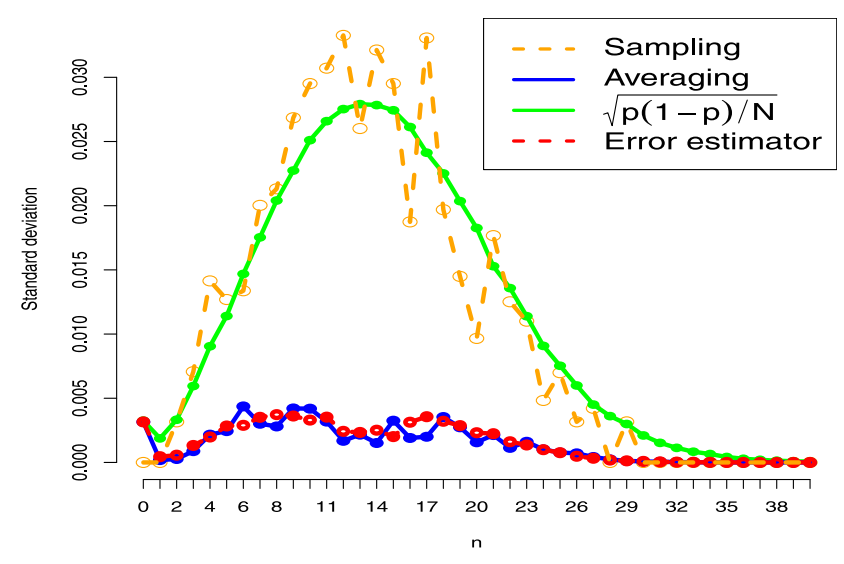

(B) Standard error

Figure 10. Distribution and standard error for the Voronoi flower complement, $\lambda=20$.

\section{Distribution of Stopping SETS}

In this section we estimate the distribution $\mathbb{P}(N(\mathrm{~S})=n)$ of the number of Poisson points inside a stopping set $\mathrm{S}$ using both the standard sampling estimator $\mathbf{1}_{\{N(\mathrm{~S})=n\}}$ and the alternative estimator

$$
\mathbf{1}_{\{N(X) \leq n\}} \frac{(-\sigma(\overline{\mathrm{S}}))^{n-N(X)}}{(n-N(X)) !} \mathrm{e}^{\sigma(\overline{\mathrm{S}})}
$$

obtained from Corollary 4.4. We note however that here, this estimator does not improve in precision over the standard sampling estimator $\mathbf{1}_{\{N(\mathrm{~S})=n\}}$.

\subsection{Annuli in finite volume}

In this setting we have $X=B(0, R), \mathrm{S}=B_{m}, h(R)=\sigma(B(0, R))$ and $\sigma(\overline{\mathrm{S}})=h(R)-\sigma\left(B_{m}\right)$, and the result of Corollary 4.4 can be recovered in closed form. 
(i) When $0 \leq l<m$, we have

$$
d \mathbb{P}\left(\sigma\left(B_{m}\right) \leq r \text { and } N(B(0, R))=l\right)=\mathbb{P}(N(B(0, R))=l) \delta_{R}(\mathrm{~d} r),
$$

and $\sigma\left(B_{m}\right)=h(R)$ if $N(X)<m$, hence the estimator (4.4) coincides with the standard sampling estimator, as

$$
\mathbf{1}_{\{N(X) \leq l\}} \frac{\left(\sigma\left(B_{m}\right)-h(R)\right)^{l-N(X)}}{(l-N(X)) !} \mathrm{e}^{h(R)-\sigma\left(B_{m}\right)}=\mathbf{1}_{\{N(X)=l\}} .
$$

(ii) When $l \geq m$, we have

$$
\begin{aligned}
& \mathbb{P}\left(\sigma\left(B_{m}\right)>h(r) \text { and } N(B(0, R))=l\right)=\mathbb{P}(N(B(0, r))<m \text { and } N(B(0, R))=l) \\
& =\mathbb{P}(N(B(0, R))-N(B(0, r))>l-m \text { and } N(B(0, r)+N(B(0, R))-N(B(0, r))=l) \\
& =\sum_{p=0}^{l} \mathbf{1}_{\{l-p>l-m\}} \mathbb{P}(N(B(0, R))-N(B(0, r))=l-p) \mathbb{P}(N(B(0, r))=p) \\
& =\mathrm{e}^{-h(R)} \sum_{p=0}^{m-1} \frac{(h(R)-h(r))^{l-p}}{(l-p) !} \frac{(h(r))^{p}}{p !}, \quad 0 \leq r<R,
\end{aligned}
$$

and

$$
\begin{aligned}
d \mathbb{P}\left(\sigma\left(B_{m}\right)\right. & \leq r \text { and } N(B(0, R))=l)=-d \mathbb{P}(N(B(0, r))<m \text { and } N(B(0, R))=l) \\
& =\mathrm{e}^{-R}\left(\sum_{p=0}^{m-1} \frac{(R-r)^{l-p-1}}{(l-p-1) !} \frac{r^{p}}{p !}-\sum_{p=1}^{m-1} \frac{(R-r)^{l-p}}{(l-p) !} \frac{r^{p-1}}{(p-1) !}\right) \mathrm{d} r \\
& =\mathrm{e}^{-R} \frac{(R-r)^{l-m}}{(l-m) !} \frac{r^{m-1}}{(m-1) !} \mathrm{d} r
\end{aligned}
$$

hence when $l=m$, the right hand side of (4.5) reads

$$
\begin{aligned}
\mathbb{E}\left[\mathbf{1}_{\{N(X) \leq m\}} \frac{(-1)^{m-N(X)}}{(m-N(X)) !}\right. & \left.\left(h(R)-\sigma\left(B_{m}\right)\right)^{m-N(X)} \mathrm{e}^{h(R)-\sigma\left(B_{m}\right)}\right]=\mathbb{E}\left[\mathbf{1}_{\{N(X)=m\}} \mathrm{e}^{h(R)-\sigma\left(B_{m}\right)}\right] \\
& =\int_{0}^{h(R)} \mathrm{e}^{-x} \frac{x^{m-1}}{(m-1) !} \mathrm{d} x \\
& =\mathbb{P}(N(X) \geq m) \\
& =\mathbb{P}\left(N\left(B_{m}\right)=m\right),
\end{aligned}
$$

while when $l>m$ we find

$$
\begin{aligned}
\mathbb{E}\left[\mathbf{1}_{\{N(X) \leq l\}}\right. & \left.\frac{(-1)^{l-N(X)}}{(l-N(X)) !}\left(h(R)-\sigma\left(B_{m}\right)\right)^{l-N(X)} \mathrm{e}^{h(R)-\sigma\left(B_{m}\right)}\right] \\
& =\sum_{k=m}^{l} \frac{(-1)^{l-k}}{(l-k) !} \mathbb{E}\left[\mathbf{1}_{\{N(X)=k\}}\left(h(R)-\sigma\left(B_{m}\right)\right)^{l-k} \mathrm{e}^{h(R)-\sigma\left(B_{m}\right)}\right] \\
& =\sum_{k=m}^{l} \frac{(-1)^{l-k}}{(l-k) !} \int_{0}^{h(R)} \mathrm{e}^{-x} \frac{(h(R)-x)^{l-m}}{(k-m) !} \frac{x^{m-1}}{(m-1) !} \mathrm{d} x
\end{aligned}
$$




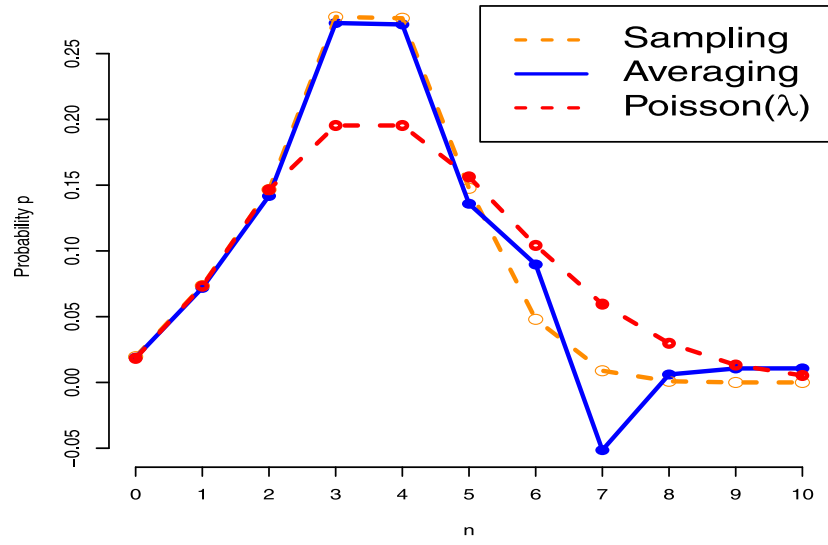

(A) Probability distribution

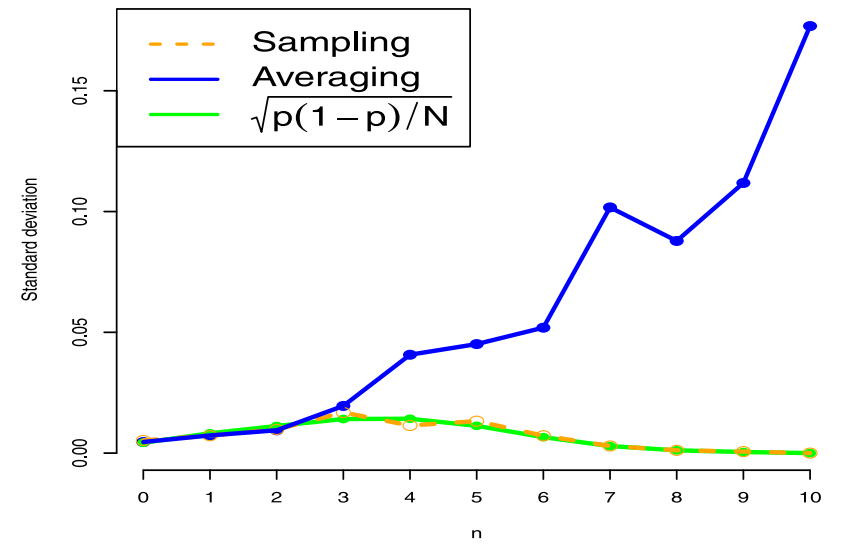

(B) Standard error

Figure 11. Distribution and standard error for the Poisson convex hull boundary, $\lambda=4$.

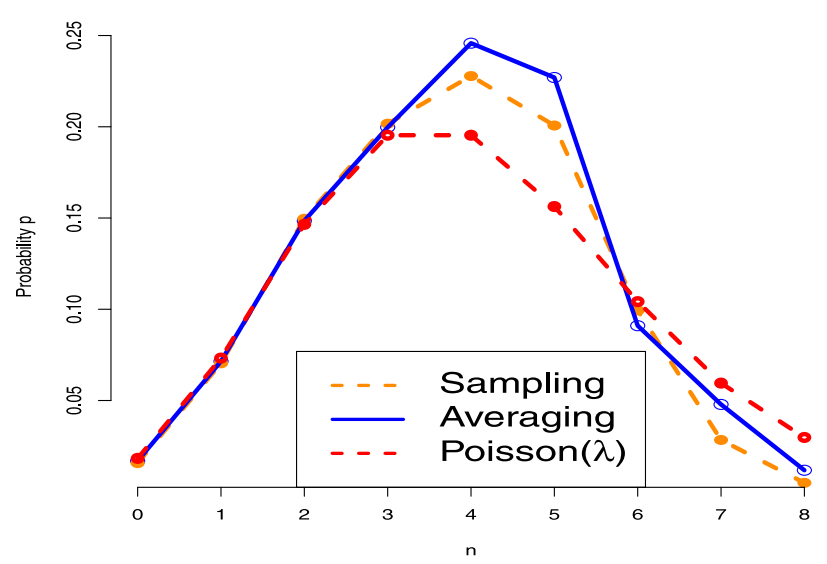

(A) Probability distribution

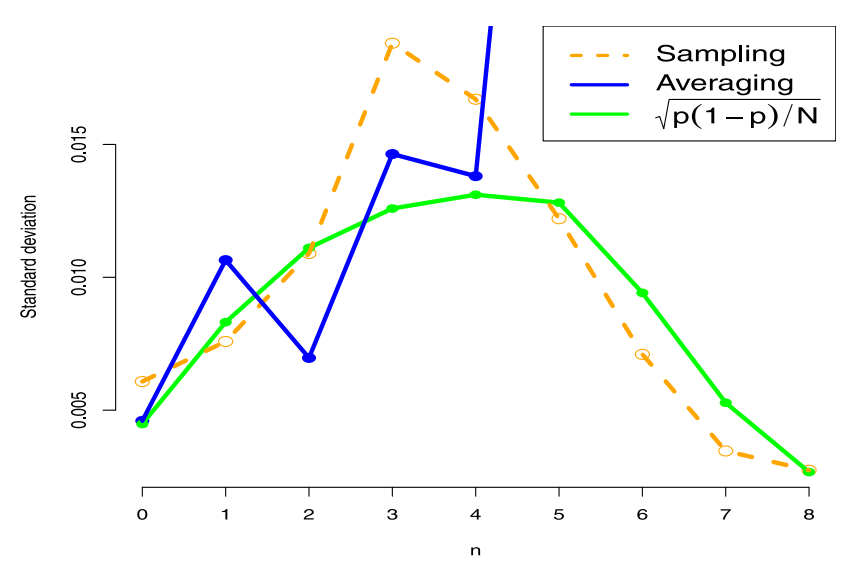

(B) Standard error

FiguRE 12. Distribution and standard error for the Voronoi flower, $\lambda=4$.

$$
\begin{aligned}
& =\int_{0}^{h(R)} \mathrm{e}^{-x}(h(R)-x)^{l-m} \frac{x^{m-1}}{(m-1) !} \mathrm{d} x \sum_{k=0}^{l-m} \frac{(-1)^{l-m-k}}{(l-m-k) ! k !} \\
& =0=\mathbb{P}\left(N\left(B_{m}\right)=l\right),
\end{aligned}
$$

which recovers the equality (4.5).

\subsection{Convex hull of a Poisson point process}

Next, we apply Corollary 4.4 to estimate the distribution $\mathbb{P}(N(\mathrm{~S})=n)$ of the count of boundary points in the convex hull of Section 5.2. Unlike in Section 5, in this example and in the next one, no particular improvement is observed when applying the estimator (6.1) ("Averaging") instead of the standard estimator $\mathbf{1}_{\{N(\mathrm{~S})=n\}}$ ("Sampling") in Figure 11, which is plotted with $N=10,000$ Monte Carlo samples. In this case, we have $\mathrm{S}=X$ when $N(X) \leq 2$, hence $\mathbb{P}(N(\mathrm{~S})=n)=\mathbb{P}(N(X)=n)$ for $n=0,1,2$. 


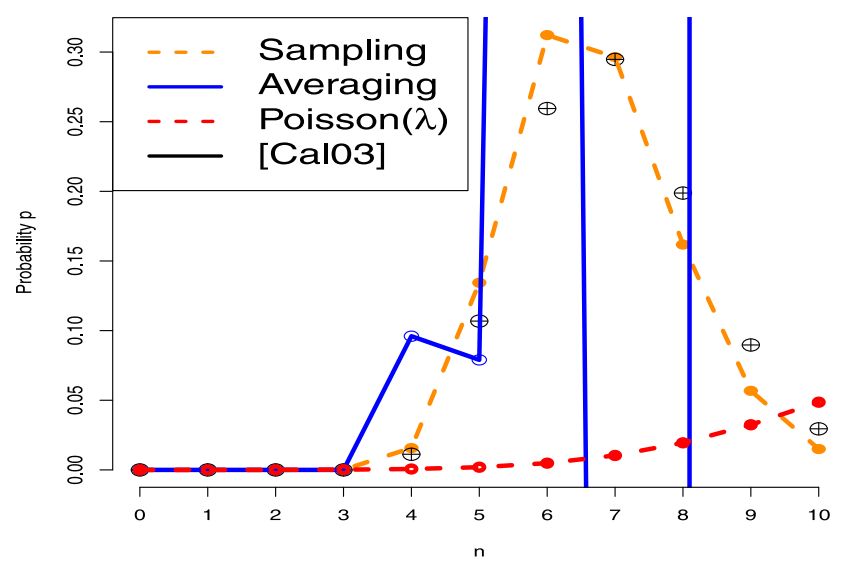

(A) $\lambda=15$

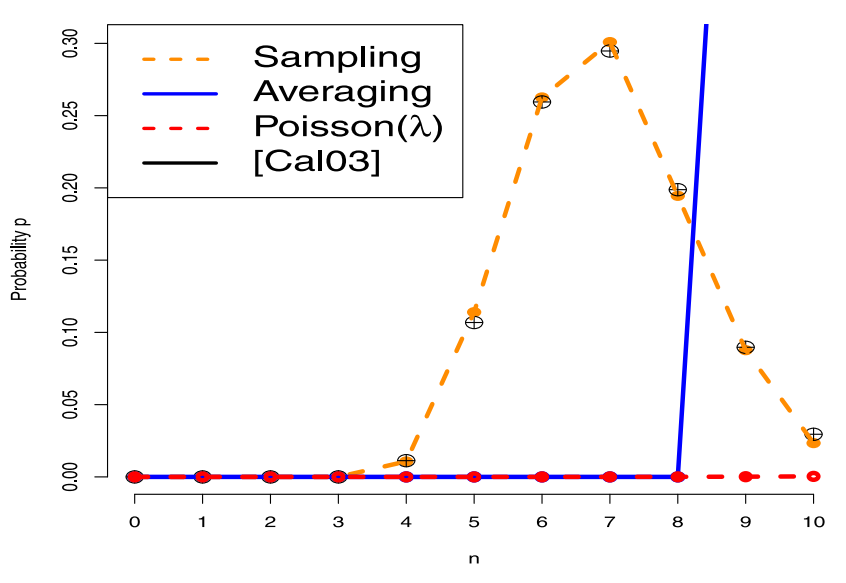

(B) $\lambda=50$

Figure 13. Distributions of the Voronoi flower for different values of $\lambda$.

Figure $11 \mathrm{~b}$ compares the variance of $\mathbf{1}_{\{N(\mathrm{~S})=n\}}$ ("Sampling") to that of (6.1) ("Averaging") when estimating the number of boundary vertices of the convex hull of a Poisson point process on the unit square $X=[0,1] \times[0,1]$. The error estimates are provided as Monte Carlo error estimates for the estimator $\mathbf{1}_{\{N(\mathrm{~S})=n\}}$ ("Sampling") together with (5.5) and (6.1) ("Averaging").

\subsection{Voronoi flower}

Here we consider the Voronoi flower S of Section 5.3 based on a typical cell containing the point $(1 / 2,1 / 2)$ in the square $X=[0,1] \times[0,1]$, up to a translation of the Poisson point process. Closed form expressions for the distribution of the number of points of the typical Voronoi cell have been obtained using the Slivnyak-Mecke identity and integration on simplexes in [5] and references therein.

Figure 12, which is plotted with $N=10,000$ Monte Carlo samples, compares the accuracy of the standard estimator $\mathbf{1}_{\{N(\mathrm{~S})=n\}}$ ("Sampling") to the estimator (6.1) ("Averaging") when estimating the count of points in the Voronoi flower $S$ around the point $(1 / 2,1 / 2)$. In this case we have $N(\mathrm{~S})=N(X)$ when $N(X) \leq 3$, and we check that $\mathbb{P}(N(\mathrm{~S})=n)=\mathbb{P}(N(X)=n)$ for $n=0,1,2,3$.

As we are dealing with a finite volume, our estimates can be compared with the distribution estimates of e.g. Table 1 in [5] for the typical Voronoi cell only when the intensity of the underlying Poisson process tends to infinity, in which case the number of points in the Voronoi flower becomes the number of points in the typical Voronoi cell plus one, see the next Figure 13 with $N=10,000$ Monte Carlo samples. However, the loss of performance of the averaging estimator (6.1) observed in Figure 12 becomes even stronger as $\lambda$ becomes large.

\section{Appendix A}

Proof of Lemma 3.4.

Let $B \in \mathcal{F}_{\mathrm{S}}$. For any compact set $K \in \mathcal{K}(X)$, by (3.2) we have

$$
B \cap\left\{\omega \in \Omega^{X}: \mathrm{S}(\omega) \subset K\right\} \in \mathcal{F}_{K},
$$

hence the random variable $\mathbf{1}_{B} \mathbf{1}_{\{\mathrm{S} \subset K\}}$ is $\mathcal{F}_{K}$-measurable, and by the definition (3.1) of $\mathcal{F}_{K}$ its value is not affected by Poisson points outside of $K$. Thus, for all $\omega \in \Omega^{X}$ and $x$ in the complement $\bar{K}$ of $K$, we find

$$
\varepsilon_{x}^{+}\left(\mathbf{1}_{B} \mathbf{1}_{\{\mathrm{S} \subset K\}}\right)(\omega)=\mathbf{1}_{B}(\omega) \mathbf{1}_{\{\mathrm{S}(\omega) \subset K\}},
$$


i.e.

$$
\mathbf{1}_{\{\mathrm{S}(\omega \cup\{x\}) \subset K\}}\left(\varepsilon_{x}^{+} \mathbf{1}_{B}\right)(\omega)=\mathbf{1}_{\{\mathrm{S}(\omega) \subset K\}} \mathbf{1}_{B}(\omega), \quad \omega \in \Omega^{X}, \quad x \in \bar{K} .
$$

In addition, since (A.1) is valid for all $\omega \in \Omega^{X}$ and $K \in \mathcal{K}(X)$, we can fix $\omega^{\prime} \in \Omega^{X}$ and apply (A.1) to a compact $K\left(\omega^{\prime}\right)$ depending on $\omega^{\prime}$, which yields

$$
\mathbf{1}_{\left\{\mathrm{S}(\omega \cup\{x\}) \subset K\left(\omega^{\prime}\right)\right\}}\left(\varepsilon_{x}^{+} \mathbf{1}_{B}\right)(\omega)=\mathbf{1}_{\left\{\mathrm{S}(\omega) \subset K\left(\omega^{\prime}\right)\right\}} \mathbf{1}_{B}(\omega), \quad \omega \in \Omega^{X}, \quad x \in \bar{K}\left(\omega^{\prime}\right),
$$

or, in the particular case where we let $\omega:=\omega^{\prime}$,

$$
\mathbf{1}_{\left\{\mathrm{S}\left(\omega^{\prime} \cup\{x\}\right) \subset K\left(\omega^{\prime}\right)\right\}}\left(\varepsilon_{x}^{+} \mathbf{1}_{B}\right)\left(\omega^{\prime}\right)=\mathbf{1}_{\left\{\mathrm{S}\left(\omega^{\prime}\right) \subset K\left(\omega^{\prime}\right)\right\}} \mathbf{1}_{B}\left(\omega^{\prime}\right), \quad \omega^{\prime} \in \Omega^{X}, \quad x \in \bar{K}\left(\omega^{\prime}\right) .
$$

Let now $\omega \in \Omega^{X}$ and $x \in \overline{\mathrm{S}}(\omega)$. Since $\mathrm{S}(\omega)$ is a closed (and compact) set in $X$, there exists $K(\omega) \in \mathcal{K}(X)$ such that

$$
x \in \bar{K}(\omega) \subset \overline{\mathrm{S}}(\omega)
$$

with

$$
\mathrm{S}(\omega \cup\{x\}) \subset \mathrm{S}(\omega) \subset K(\omega)
$$

since $\mathrm{S}(\omega)$ is non-increasing. Hence, by (A.2) we have

$$
\begin{aligned}
\varepsilon_{x}^{+} \mathbf{1}_{B}(\omega) & =\mathbf{1}_{\{\mathrm{S}(\omega \cup\{x\}) \subset K(\omega)\}} \varepsilon_{x}^{+} \mathbf{1}_{B}(\omega) \\
& =\mathbf{1}_{\{\mathrm{S}(\omega) \subset K(\omega)\}} \mathbf{1}_{B}(\omega) \\
& =\mathbf{1}_{B}(\omega), \quad x \in \overline{\mathrm{S}}(\omega) .
\end{aligned}
$$

This conclusion extends from $B \in \mathcal{F}_{\mathrm{S}}$ to any $\mathcal{F}_{\mathrm{S}}$-measurable random variable $F(\omega)$ by a monotone class argument.

Proof of Proposition 3.5. (See also Prop. 3.3 of [4]).

Let $x_{1}, \ldots, x_{n} \in X$. We consider the following cases.

(i) If $\left\{x_{1}, \ldots, x_{n}\right\} \subset \overline{\mathrm{S}}(\omega)$ then we have $\left\{x_{1}, \ldots, x_{n}\right\} \subset \overline{\mathrm{S}}\left(\omega \cup\left\{x_{1}, \ldots, x_{n}\right\}\right)$ because $\overline{\mathrm{S}}(\omega)$ is non-decreasing, hence by Lemma 3.4 we have

$$
\varepsilon_{\mathfrak{x}_{n}}^{+}\left(\mathbf{1}_{\overline{\mathrm{S}}}\left(x_{1}\right) \cdots \mathbf{1}_{\overline{\mathrm{S}}}\left(x_{n}\right)\right)=\mathbf{1}_{\overline{\mathrm{S}}}\left(x_{1}\right) \cdots \mathbf{1}_{\overline{\mathrm{S}}}\left(x_{n}\right)=1 .
$$

(ii) In case $\left\{x_{1}, \ldots, x_{n}\right\} \subset \mathrm{S}(\omega)$, it follows from Lemma A.1 below that there exists $x_{e} \in\left\{x_{1}, \ldots, x_{n}\right\}$ such that $x_{e} \in \mathrm{S}\left(\omega \cup\left\{x_{1}, \ldots, x_{n}\right\}\right)$, hence

$$
\varepsilon_{\mathfrak{x}_{n}}^{+}\left(\mathbf{1}_{\overline{\mathrm{S}}}\left(x_{1}\right) \cdots \mathbf{1}_{\overline{\mathrm{S}}}\left(x_{n}\right)\right)=\mathbf{1}_{\overline{\mathrm{S}}}\left(x_{1}\right) \cdots \mathbf{1}_{\overline{\mathrm{S}}}\left(x_{n}\right)=0 .
$$

(iii) If $\left\{x_{1}, \ldots, x_{n}\right\} \cap \mathrm{S}(\omega) \neq \emptyset$ we partition $\left\{x_{1}, \ldots, x_{n}\right\}$ as

$$
\left\{x_{1}, \ldots, x_{n}\right\}=\left\{x_{1}, \ldots, x_{k}\right\} \cup\left\{x_{k+1}, \ldots, x_{n}\right\}
$$

with $\left\{x_{1}, \ldots, x_{k}\right\} \subset \mathrm{S}(\omega)$ and $\left\{x_{k+1}, \ldots, x_{n}\right\} \subset \overline{\mathrm{S}}(\omega)$, for some $k \in\{1, \ldots, n\}$. By point $(i i)$ above we have

$$
\varepsilon_{\mathfrak{x}_{k}}^{+}\left(\mathbf{1}_{\overline{\mathrm{S}}}\left(x_{1}\right) \cdots \mathbf{1}_{\overline{\mathrm{S}}}\left(x_{k}\right)\right)=\mathbf{1}_{\overline{\mathrm{S}}}\left(x_{1}\right) \cdots \mathbf{1}_{\overline{\mathrm{S}}}\left(x_{k}\right)=0
$$


and by Lemma 3.4 we have $\overline{\mathrm{S}}(\omega)=\overline{\mathrm{S}}\left(\omega \cup\left\{x_{k+1}, \ldots, x_{n}\right\}\right)$, hence

$$
\begin{aligned}
\varepsilon_{\mathfrak{x}_{n}}^{+}\left(\mathbf{1}_{\overline{\mathrm{S}}}\left(x_{1}\right) \cdots \mathbf{1}_{\overline{\mathrm{S}}}\left(x_{n}\right)\right) & =\varepsilon_{\mathfrak{x}_{n}}^{+}\left(\mathbf{1}_{\overline{\mathrm{S}}}\left(x_{1}\right) \cdots \mathbf{1}_{\overline{\mathrm{S}}}\left(x_{k}\right)\right) \varepsilon_{\mathfrak{x}_{n}}^{+}\left(\mathbf{1}_{\overline{\mathrm{S}}}\left(x_{k+1}\right) \cdots \mathbf{1}_{\overline{\mathrm{S}}}\left(x_{n}\right)\right) \\
& =\varepsilon_{\mathfrak{x}_{k}}^{+}\left(\mathbf{1}_{\overline{\mathrm{S}}}\left(x_{1}\right) \cdots \mathbf{1}_{\overline{\mathrm{S}}}\left(x_{k}\right)\right) \varepsilon_{\mathfrak{x}_{k}}^{+}\left(\mathbf{1}_{\overline{\mathrm{S}}}\left(x_{k+1}\right) \cdots \mathbf{1}_{\overline{\mathrm{S}}}\left(x_{n}\right)\right) \\
& =\mathbf{1}_{\overline{\mathrm{S}}}\left(x_{1}\right) \cdots \mathbf{1}_{\overline{\mathrm{S}}}\left(x_{k}\right) \varepsilon_{\mathfrak{x}_{k}}^{+}\left(\mathbf{1}_{\overline{\mathrm{S}}}\left(x_{k+1}\right) \cdots \mathbf{1}_{\overline{\mathrm{S}}}\left(x_{n}\right)\right) \\
& =0 \\
& =\mathbf{1}_{\overline{\mathrm{S}}}\left(x_{1}\right) \cdots \mathbf{1}_{\overline{\mathrm{S}}}\left(x_{n}\right), \quad \mathfrak{x}_{k}=\left(x_{1}, \ldots, x_{k}\right) .
\end{aligned}
$$

The next lemma has been used in the proof of Proposition 3.5.

Lemma A.1. Let $\mathrm{S}$ be a stable and non-increasing stopping set. For any $\omega \in \Omega^{X}$, and $x_{1}, \ldots, x_{k} \in \mathrm{S}(\omega)$, there exists $i \in\{1, \ldots, k\}$ such that $x_{i} \in \mathrm{S}\left(\omega \cup\left\{x_{1}, \ldots, x_{k}\right\}\right)$.

Proof. We do the proof by contradiction by assuming that $\left\{x_{1}, \ldots, x_{k}\right\} \subset \overline{\mathrm{S}}\left(\omega \cup\left\{x_{1}, \ldots, x_{k}\right\}\right)$. We will show by induction on $j=1, \ldots, k+1$ that

$$
\overline{\mathrm{S}}\left(\omega \cup\left\{x_{1}, \ldots, x_{k}\right\}\right)=\overline{\mathrm{S}}\left(\omega \cup \bigcup_{i=j}^{k}\left\{x_{i}\right\}\right),
$$

with the convention $\cup_{i=k+1}^{k}\left\{x_{i}\right\}=\emptyset$. For $j=k+1$ this leads to $\overline{\mathrm{S}}\left(\omega \cup\left\{x_{1}, \ldots, x_{k}\right\}\right)=\overline{\mathrm{S}}(\omega)$ and to $x_{j} \in \overline{\mathrm{S}}(\omega)$, $j=1, \ldots, k$, which contradicts $\left\{x_{1}, \ldots, x_{k}\right\} \subset \mathrm{S}(\omega)$.

Relation (A.3) clearly holds for $j=1$, and we suppose that it holds for some $j \in\{1, \ldots, k\}$. By assumption we have

$$
x_{j} \in \overline{\mathrm{S}}\left(\omega \cup\left\{x_{1}, \ldots, x_{k}\right\}\right)=\overline{\mathrm{S}}\left(\omega \cup \cup_{i=j}^{k}\left\{x_{i}\right\}\right),
$$

hence

$$
x_{j} \in \overline{\mathrm{S}}\left(\omega \cup \cup_{i=j+1}^{k}\left\{x_{i}\right\}\right)
$$

by the stability condition (3.3). Consequently, by (3.4) or Lemma 3.4 we have

$$
\overline{\mathrm{S}}\left(\omega \cup\left\{x_{j+1}, \ldots, x_{k}\right\}\right)=\overline{\mathrm{S}}\left(\omega \cup \bigcup_{i=j}^{k}\left\{x_{i}\right\}\right)
$$

since $\mathrm{S}(\omega)$ is a stable and non-increasing stopping set.

\section{REFERENCES}

[1] P.K. Agarwal, S. Har-Peled, S. Suri, H. Yılıdz and W. Zhang, Convex hulls under uncertainty. Algorithmica 79 (2017) $340-367$.

[2] A. Baddeley and R. Turner, spatstat: An R package for analyzing spatial point patterns. J. Stat. Softw. 12 (2005) 1-42.

[3] N. Baldin and M. Reiß, Unbiased estimation of the volume of a convex body. Stochastic Process. Appl. 126 (2016) 3716-3732.

[4] J.-C. Breton and N. Privault, Factorial moments of point processes. Stochastic Process. Appl. 124 (2014) $3412-3428$.

[5] P. Calka, An explicit expression for the distribution of the number of sides of the typical Poisson-Voronoi cell. Adv. Appl. Probab. 35 (2003) 863-870.

[6] R. Cowan, A more comprehensive complementary theorem for the analysis of Poisson point processes. Adv. Appl. Probab. 38 (2006) 581-601. 
[7] R. Cowan, M. Quine and S. Zuyev, Decomposition of gamma-distributed domains constructed from Poisson point processes. Adv. Appl. Probab. 35 (2003) 56-69.

[8] Y. Davydov and S. Nagaev, On the convex hulls of point processes. Manuscript (2000).

[9] L. Decreusefond and I. Flint, Moment formulae for general point processes. J. Funct. Anal. 267 (2014) $452-476$.

[10] T.G. Kurtz, The optional sampling theorem for martingales indexed by directed sets. Ann. Probab. 8 (1980) 675-681.

[11] J. Mecke, Stationäre zufällige Masse auf lokalkompakten Abelschen Gruppen. Z. Wahrscheinlichkeitstheorie Verw. Geb. 9 (1967) 36-58.

[12] R.E. Miles, On the homogeneous planar Poisson point process. Math. Biosci. 6 (1970) 85-127.

[13] I. Molchanov, Theory of random sets. Probability and its Applications. Springer-Verlag, London, New York (2005).

[14] X.X. Nguyen and H. Zessin, Integral and differential characterization of the Gibbs process. Math. Nachr. 88 (1979) $105-115$.

[15] N. Privault, Moment identities for Poisson-Skorohod integrals and application to measure invariance. C. R. Math. Acad. Sci. Paris 347 (2009) 1071-1074.

[16] N. Privault, Invariance of Poisson measures under random transformations. Ann. Inst. Henri Poincaré Probab. Statist. 48 (2012) 947-972.

[17] N. Privault, Moments of Poisson stochastic integrals with random integrands. Prob. Math. Stat. 32 (2012) $227-239$.

[18] N. Privault, Laplace transform identities for the volume of stopping sets based on Poisson point processes. Adv. Appl. Probab. 47 (2015) 919-933.

[19] R. Schneider and W. Weil, Stochastic and integral geometry. Probability and its Applications. Springer-Verlag, Berlin, New York (2008).

[20] I.M. Slivnyak, Some properties of stationary flows of homogeneous random events. Theory Probab. Appl. 7 (1962) 336-341.

[21] S. Zuyev, Stopping sets: gamma-type results and hitting properties. Adv. Appl. Probab. 31 (1999) 355-366. 